\title{
Lomonosov GRB Catalogue: The First Experience of Prompt Emission Multi-Wavelength Observations
}

\author{
V. A. Sadovnichy ${ }^{1}$, M. I. Panasyuk 1,2, S. I. Svertilov 1,2,*, V. M. Lipunov 2,3, V. V. Bogomolov ${ }^{1,2}$, \\ A. V. Bogomolov ${ }^{1, *}$, E. S. Gorbovskoy ${ }^{3}$, A. F. Iyudin ${ }^{1}$, V. V. Kalegaev ${ }^{1}$, V. G. Kornilov ${ }^{2,3}$, I. H. Park ${ }^{4}$ (D), \\ V. L. Petrov ${ }^{1}$, N. N. Vedenkin ${ }^{4}$ and I. V. Yashin ${ }^{1}$
}

check for

updates

Citation: Sadovnichy, V.A.;

Panasyuk, M.I.; Svertilov, S.I.;

Lipunov, V.M.; Bogomolov, V.V.;

Bogomolov, A.V.; Gorbovskoy, E.S.;

Iyudin, A.F.; Kalegaev, V.V.; Kornilov,

V.G.; et al. Lomonosov GRB

Catalogue: The First Experience of

Prompt Emission Multi-Wavelength Observations. Universe 2021, 7, 375.

https://doi.org/10.3390/

universe7100375

Academic Editors: Jakub Ripa and

Mariusz Tarnopolski

Received: 6 September 2021

Accepted: 3 October 2021

Published: 9 October 2021

Publisher's Note: MDPI stays neutral with regard to jurisdictional claims in published maps and institutional affiliations.

Copyright: (c) 2021 by the authors. Licensee MDPI, Basel, Switzerland This article is an open access article distributed under the terms and conditions of the Creative Commons Attribution (CC BY) license (https:// creativecommons.org/licenses/by/ $4.0 /)$.
1 Skobeltsyn Institute of Nuclear Physics, Lomonosov Moscow State University, 119234 Moscow, Russia; info@rector.msu.ru (V.A.S.); panasyuk@sinp.msu.ru (M.I.P.); bogovit@rambler.ru (V.V.B.); aiyudin@srd.sinp.msu.ru (A.F.I.); klg@dec1.sinp.msu.ru (V.V.K.); vas.i.nas@gmail.com (V.L.P.); ivn@eas.sinp.msu.ru (I.V.Y.)

2 Department of Physics, Lomonosov Moscow State University, 119234 Moscow, Russia; lipunov2007@gmai.com (V.M.L.); kornilov@sai.msu.ru (V.G.K.)

3 Sternberg Astronomical Institute, Lomonosov Moscow State University, 119234 Moscow, Russia; gorbovskoy@sai.msu.ru

4 Department of Physics, Sungkyunkwan University, 2066 Seobu-ro, Jangan-gu, Gyeong Gi-do, Suwon-si 16419, Korea; ilpark@skku.edu (I.H.P.); vnn@soace.gmail.com (N.N.V.)

* Correspondence: sis@coronas.ru (S.I.S.); aabboogg@sinp.msu.ru (A.V.B.)

Abstract: This paper presents a catalogue of gamma-ray bursts (GRBs) that were detected by the instruments onboard the Lomonosov space observatory. The Lomonosov mission gave the first experience of not only multi-wavelength (from optical to gamma) observations of GRBs but also multi-messenger observations of extreme phenomena and GRBs. The detailed light curves and energy spectra of the detected GRBs are presented. The results of the prompt, early an afterglow optical observations of several GRBs are discussed.

Keywords: gamma-ray bursts; prompt; afterglow emission; multi-wavelength; multi-messenger observations

\section{Introduction}

One of the most important and interesting branches of modern physics and astrophysics is the study of extreme processes and phenomena that lead to the most powerful explosions and acceleration of particles to the maximum possible energies. Such phenomena include ultra-high-energy cosmic rays (UHECR) and cosmic gamma-ray bursts (GRBs hereafter), i.e., long-term phenomena that are associated with the collapse of massive stars (hypernovae) and short-term, transient phenomena that are due to the merging of relativistic compact objects (neutron stars and black holes), which also leads to gravitational wave generation. The acceleration of particles to high and/or extremely high energies, as well as the generation of neutrinos of high and ultrahigh energies, can also occur in GRB sources. Although these extreme phenomena have been studied very intensively in recent years, there are still many unsolved problems, where the effective ways of solving these problems involve using multi-channel and multi-wavelength observations.

The scientific importance of solving the problem of extremely high energy particle acceleration via the study of UHECR and GRBs means the ability to determine the maximum energy of particle acceleration in astrophysical sources, the acceleration mechanisms and the nature of these sources. Evidence of the acceleration of UHECR in GRB sources will be crucial to confirm the GZK effect and thus verify the adequacy of cosmological models. Regarding progress in understanding the nature of GRBs, the relevance and importance of this research confirmed the exceptional importance of the role played by 
GRBs in astrophysics as the most powerful events in the Universe, which can also be used as an independent test of cosmological models.

As it is well known, GRBs are the most powerful explosions in the Universe, where the power of their sources can reach $10^{54} \mathrm{erg} / \mathrm{s}$. GRBs are characterised by a wide variety of temporal structures. Their duration can range from fractions of a second to tens or even hundreds of seconds. It is customary to distinguish two populations of GRBs, so-called short (lasting less than a few seconds) and long, with a duration from seconds to hundreds of seconds. The energy spectra of GRBs have a decreasing character, where the values of the typical energies lie in the range of $0.1-1.0 \mathrm{MeV}$, and short bursts are characterised by harder spectra than long-term ones. Experiments on observing the optical afterglow of GRBs showed that the sources of at least long GRBs are located in distant galaxies at very high redshifts. Apparently, today, the GRB sources are characterised by the highest redshift values among extragalactic objects [1]. Therefore, the study of GRBs can provide both unique information about the early stages of the Universe's evolution and about the physical processes occurring at such energy densities that are principally unattainable in ground-based laboratories.

Modern ideas about the nature of long GRBs suggest that they are caused by the collapse of supermassive stars, the so-called hypernovae. It is assumed that such supermassive stars are born and evolve mainly at the early stages of primary star formation, which is confirmed by the fact that the redshifts $z$ of GRB sources obtained from optical afterglow can reach values of $z \sim 10$. Therefore, we can say that the GRB distribution on $z$ reflects the history of primary star formation, including in the epoch of the so-called reionisation $(z \sim 7)$. It is very reasonable to expand the range of observed $z$ values to about 15 , which will make it possible to obtain information about the epoch that included the formation of the first stars and galaxies in the Universe [2]. GRBs can also be used as an independent cosmological test [3-6], operating in a significantly wider range of redshifts in comparison with traditional cosmological tests based on observations of type Ia supernovae [7], baryon oscillations [8] and gravitational lensing [9]. Furthermore, of particular interest is the study of so-called dark GRBs, which can provide information about events that are invisible in optics due to the absorption on the so-called "forest" of Lyman-alpha lines [10].

The other problems in the study of GRBs are their use for the research of extreme processes in astrophysical objects. First of all, they provide an understanding of the mechanism of generation of the most powerful explosions in the Universe, which are the GRBs, including the "central machine" operation details. Since relativistic shock waves arising during its operation are very efficient accelerators, sources of neutrinos [11] could be associated with the GRBs. Short gamma-ray bursts are associated with the merging of neutron stars and black holes; such processes can also lead to the generation of gravitational waves [12]. Therefore, simultaneous observations of GRBs by orbital instruments, as well as the detection of neutrinos (e.g., ICECUBE [13], ANITA [14]) and gravitational waves (e.g., LIGO [15], LISA/NGO [16]), are an example of the effectiveness of multichannel astronomy. Ground-based (e.g., TA [17,18], Auger [19]) and orbital (TUS [20], JEM-EUSO [21]) installations for UHECR can give additional data, especially if they are capable of detecting UHE neutrinos.

The GRB study is also a good example of successful multi-wavelength observations. Since the detection of optical afterglow, their sources were identified as distant, so-called host galaxies, where the observation of the afterglow in UV and optics is the main way to determine the distance to their sources [22]. The time lag $\Delta t_{\text {lag }}$ between the detection of the burst in hard X-rays and gamma rays and the observation of the optical afterglow is determined mainly by the time of reorientation of the optical telescope according to the target designation of the gamma detector. Among the orbital experiments, the smallest time was achieved at the Swift space observatory and is on the order of tens of minutes [23]. For large ground-based telescopes, the $\Delta t_{\text {lag }}$ value is much larger and can reach several hours. It is only possible for robotic telescopes of the MASTER network (see below) to reduce this value down to several minutes. However, given the necessity of GRB prompt 
emission detection in optics, as well as of the so-called precursor detection, reducing the time lag becomes a very important task. This is necessary for plotting spectra in a wide range of wavelengths, which can provide key information about the mechanism of the "central engine". A separate problem is the search for optical and UV partners for short GRBs, where the number of observations in optics of these is currently extremely limited. All this requires reducing the $\Delta t_{\text {lag }}$ value to dozens of seconds or less. This requires new tools and new approaches to organising observations, both in orbital and ground-based experiments.

Observations of GRBs in optical and gamma ranges that do not require a redirection of optical instruments are first made on the Lomonosov satellite. This is achieved with the use of wide-field optical cameras, which are installed coaxially with the gamma detectors and continuous image recording is performed. Thus, it is possible not only to fix the optical light curve of gamma-ray burst prompt emissions but also to detect the precursors in the optical range.

\section{2. "Lomonosov" Space Mission}

The Russian space mission Lomonosov (http://lomonosov.sinp.msu.ru, accessed on 4 September 2021) was launched on 28.04.2016 from "Vostochny" to a polar solarsynchronous orbit with an altitude of $\sim 490 \mathrm{~km}$. The scientific instruments on board the Lomonosov include the TUS instrument for viewing tracks of UHECR extensive air showers (EAS) ionisation glow [24], as well as several instruments to study GRBs, including the $X$-ray and gamma-ray detector BDRG [25], wide-field optical cameras SHOK [26] and ultraviolet (UV) and X-ray telescope UFFO consisting of a UV slewing mirror telescope SMT and an X-ray imaging telescope UBAT UV slewing mirror telescope SMT [27,28]. All instruments were operated in monitor mode for continuous measurements; therefore, it is possible to simultaneously detect events in the optical, UV, X-ray and gamma ranges. Thus, the scientific program of "Lomonosov" includes a multi-wavelength GRB study at different time scales.

In most of the previous experiments, multi-wavelength observations were carried out by generating a trigger from a gamma detector and then pointing optical and soft $X$-ray telescopes into the sky region with the coordinates recorded in this trigger. Several methods are used to provide the observation of the early phase of GRB source activity. First, in the experiments of Fermi/GBM, Swift/BAT and some others, a fast automatic transfer of a message with the coordinates of a gamma-ray burst from space instruments to ground-based telescopes was realised [29]. In this case, the possibility of multi-wavelength observations is determined by the speed of pointing the ground telescope to the source. Another approach that was realised in the Swift mission is fast re-pointing of all instruments by rotating the satellite after a GRB is triggered. The time of the satellite reorientation of about several tens of seconds allows one to study GRB afterglows at early stages [29], but the very beginning phase of the GRB cannot be observed using this method.

On the Lomonosov satellite, a fundamentally different approach is used for multiwavelength observations, which does not require telescope pointing or the rotation of the spacecraft. Simultaneous observations of GRBs in the optical and gamma ranges are possible due to the real-time recording of a sequence of images from cameras with a wide field of view. It is assumed that some of the burst sources will be located in the field of view of the optical telescope. Real-time GRB data were transferred to the ground via a GlobalStar modem in the GCN network. It also allows for control events from the ground facilities and detecting UHECR (Auger, TA), high-energy neutrinos (IceCube, ANTARES) and gravitational waves (LIGO) to be realised. During the project, real-time analysis of data from the Lomonosov satellite instruments was carried out, as well as the joint analysis with data from ground-based instruments and other space experiments on GRBs, such as the space observatories from Fermi and Swift.

In particular, we note the possibilities of joint analysis of data obtained from Lomonosov and from ground-based robotic telescopes of the MASTER Global Robotic Net, which was 
developed by MSU. The MASTER robotic telescopes allow for observations of the prompt optical emission and optical afterglows of GRBs, as well as other transient astrophysical phenomena. Joint analysis of the satellite data and the data of ground robotic telescopes are very useful, as well as the operative control of BDRG gamma-ray data on triggers from ground-based facilities, including neutrino and gravitation wave detectors. For more information on the MASTER robotic telescope network, see below.

\subsection{Gamma-Ray Observations}

The instruments BDRG and UFFO were used for gamma-ray observations on board the Lomonosov satellite. The BDRG/Lomonosov [25] instrument was designed to detect GRBs and to provide measurements of the light curves and spectra of GRBs in the $10-3000 \mathrm{keV}$ energy range. The GRB triggers were produced using BDRG on $10 \mathrm{~ms}, 1 \mathrm{~s}$ and $20 \mathrm{~s}$ time scales. The triggers were provided for ground and space telescopes, including the SHOK optical cameras and the UFFO instrument. The UFFO instrument consists of the codedmask hard X-ray telescope UBAT $[27,28]$ and the fast optical telescope SMT. The design with a moving mirror allows the SMT to be pointed to the GRB source for 1 s [30,31].

During the Lomonosov mission, GRBs were detected mainly by the BDRG instrument. Three identical detector boxes, namely, BDRG-1, BDRG-2 and BDRG-3, are connected to a data analysis box BA-BDRG. The axes of the BDRG detectors are oriented $90^{\circ}$ to each other. Therefore, a GRB source can be localised in the sky with an accuracy of $\sim 2^{\circ}$ (for bright GRBs) via a comparison of the readings of the BDRG detectors. One must take into account that the detectors have a cosine angular dependence for a sensitive area. The central axis of the group of BDRG detectors is directed towards the local zenith. Thus, the total field of view (FOV) of the device is about $2 \pi$ sr. Within the solid angle $\pi / 2 \mathrm{sr}$, the position of the source can be estimated with sufficiently high accuracy. The FOVs of two BDRG detectors overlap the FOVs of SHOK optical cameras (see Figure 1a). This provides optical observations of a GRB source that is detected by BDRG without redirection of the optical instrument.

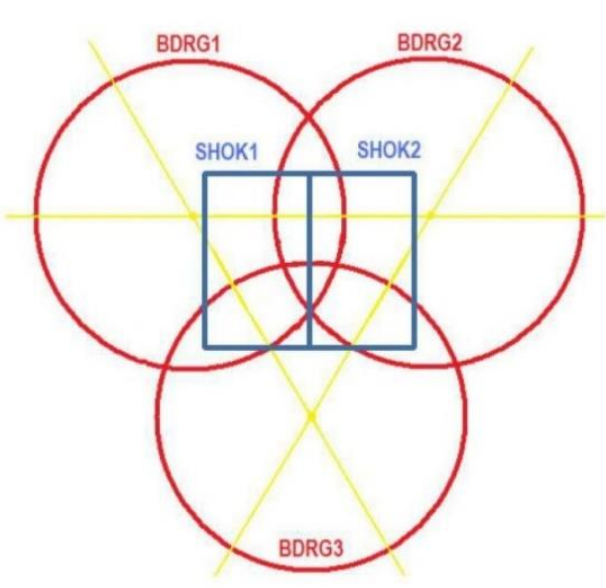

(a)

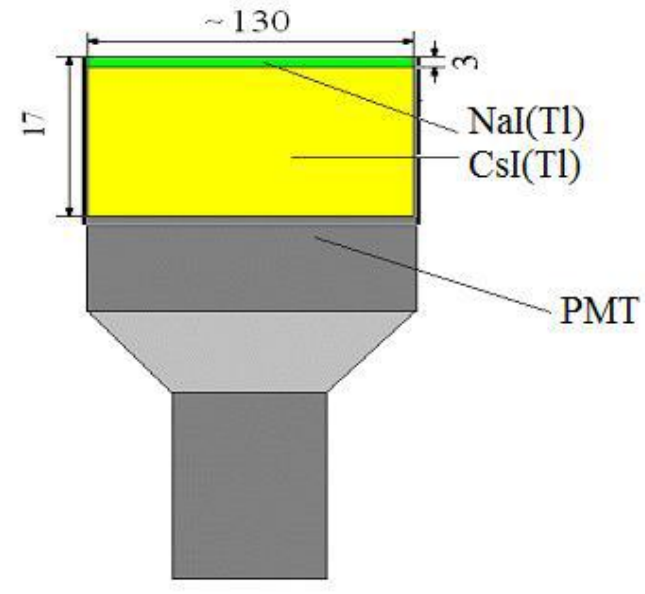

(b)

Figure 1. (a) FOV of BDRG gamma detectors, together with the FOV of SHOK optical cameras. (b) The design of the scintillation detector of the BDRG instrument.

The data from three gamma detectors are collected in a single BA BDRG box for onboard data analysis to produce a set of output data frames and GRB triggers. The daily amount of scientific data is $\sim 500 \mathrm{Mb}$. About $180 \mathrm{Mb}$ of continuous data is produced per day, and $\sim 5 \mathrm{Mb}$ of detailed information is collected for each burst trigger. Continuous data contain the count rates with a $100 \mathrm{~ms}$ time resolution (16 channels for each detector) and detailed energy spectra. A large portion of the information that is recorded in the gamma 
range by the gamma mode is collected for a trigger, with up to $10^{6}$ events for each one. The BDRG instrument sensitivity is about $10^{-7} \mathrm{erg} / \mathrm{cm}^{2}$.

The BDRG instrument parameters are given in Table 1. The structure and amount of the scientific data are presented in Table 2.

Table 1. Parameters of the BDRG instrument.

\begin{tabular}{cc}
\hline Parameter, Units & Value \\
\hline Energy range, $\mathrm{MeV}$ & $0.01-3.0$ \\
Effective area (for three detectors), $\mathrm{cm}^{2}$ & $\sim 360$ \\
Time resolution, ms & 1 (for the burst mode) \\
Field of view, sr & $2 \pi$ \\
Field of the effective source location. $\mathrm{sr}$ & $\pi / 2$ \\
Sensitivity to the burst detection, erg $/ \mathrm{cm}^{2}$ & $\sim 10^{-7}$ \\
Accuracy of burst source location & $\sim 2-4^{\circ}$ (for brightest events) \\
\hline
\end{tabular}

Table 2. Structure and amount of BDRG output data.

\begin{tabular}{ccc}
\hline Type of Frame & $\begin{array}{c}\text { Time Interval between } \\
\text { Frames }\end{array}$ & Data Amount, $\mathbf{M b}$ \\
\hline & Continuous (180 Mb per day) & \\
\hline Monitoring & $100 \mathrm{~ms}$ & 87 \\
Spectrum & $15 \mathrm{~s}$ & 48 \\
Event mode & $15 \mathrm{~s}$ & 50 \\
\hline & Burst mode for fast/slow bursts (5 Mb per burst) & \\
\hline Monitoring & $1-10 \mathrm{~ms}$ & 1.6 \\
Spectrum & $1-10 \mathrm{~s}$ & 1.6 \\
Event mode & Not regular, up to $10^{6}$ events & 1.6 \\
\hline
\end{tabular}

Each BDRG detector consists of a $0.3 \mathrm{~cm}$ layer of $\mathrm{NaI}(\mathrm{Tl})$ that is optically coupled to a $1.7 \mathrm{~cm}$ layer of $\mathrm{CsI}(\mathrm{Tl})$ (see Figure 1, right). Both layers have a diameter of $\sim 13 \mathrm{~cm}$; therefore, the detector area is about $130 \mathrm{~cm}^{2}$. Scintillators are read out using a single PMT. The $\mathrm{NaI}(\mathrm{Tl})$ layer is optimised for the $0.01-0.5 \mathrm{MeV}$ energy range and the working range of CsI(Tl) part of the detector is $0.05-3 \mathrm{MeV}$. The $\mathrm{NaI}(\mathrm{Tl})$ layer is used for hard X-ray timing, while the $\mathrm{CsI}(\mathrm{Tl})$ crystals detect gamma rays with energies up to a few $\mathrm{MeV}$ while also being an active shield against background gamma rays. The separation of the events in the scintillators is provided by special electronic circuits that analyse the pulse shapes.

BDRG data consist of a number of the data frames that are continuously generated, as well as irregularly by several types of triggers (so-called burst mode). The continuous data stream includes monitoring, spectrum and event streams, which are formed independently. Monitoring frames contain count rates in eight energy channels for each of the $\mathrm{NaI}(\mathrm{Tl})$ and CsI(Tl) scintillator layers for each of three BDRG detector units, while spectrum frames contain separate 724 channel spectra for $\mathrm{NaI}(\mathrm{Tl})$ and $\mathrm{CsI}(\mathrm{Tl})$. Event frames contain the primary data for a fixed number of detected gamma quanta, including values for energy release within the $\mathrm{NaI}(\mathrm{Tl})$ and $\mathrm{CsI}(\mathrm{Tl})$ parts of the detector, which are combined with time data. Likewise, information about all of the produced GRB triggers is stored and transferred in the form of "trigger logs", which contain the time measurement and a set of the main parameters that are calculated for the trigger. Three trigger types with characteristic times of $10 \mathrm{~ms}, 1 \mathrm{~s}$ and $20 \mathrm{~s}$ are categorised as "fast", "slow" and "super-slow", respectively. Three data frame sequences for the monitoring, spectrum and event are generated by the trigger in a manner that is similar to the continuous mode discussed above. A portion of the detailed data collected before the trigger is always included.

The data obtained after a trigger are further analysed onboard; when a trigger is identified as a GRB-like event, data fixation in the SHOK optical cameras takes place, 
including some portion of the optical data before the trigger. It allows for the capture of GRB optical light curves, not only for prompt emissions but also for their precursors. The estimation of GRB positions takes place after the BDRG trigger, and the trigger data, together with the calculated coordinates of the source, are transferred not only to the Ultrafast Flash Observatory [23] on board the Lomonosov spacecraft but also to a ground server via the Global Star transmitter and then to several telescopes through the Gamma-ray Coordinates Network (GCN).

The GRB trigger is produced independently for each BDRG detector. Several conditions for trigger production are necessary:

- A significant fast rise of hard X-ray readings must appear (25-100 keV energy release in $\mathrm{NaI}(\mathrm{Tl})$ is used for the analysis by default);

- The count rate in the hard X-ray channel must not be too high;

- Additional condition based on $\mathrm{NaI}(\mathrm{Tl}) / \mathrm{CsI}(\mathrm{Tl})$ rate ratio in different energy channels is used for excluding GRB imitation by electrons.

Several kinds of detailed data were formed for the triggers that can be produced independently on different time scales:

1. $10 \mathrm{~ms}$ scale: $1 \mathrm{~ms}$ resolution monitoring data and gamma-by-gamma data added;

2. $1 \mathrm{~s}$ scale: $10 \mathrm{~ms}$ resolution monitoring data and gamma-by-gamma data added;

3. $20 \mathrm{~s}$ scale: burst data in gamma by gamma mode added.

The comparison of three BDRG detector readings is used to estimate the GRB coordinates. Considering that these detectors have a cosine beam of view and their axes are shifted at $90^{\circ}$ from each other, one can use the formula $\cos \theta_{\mathrm{i}}=\mathrm{N}_{\mathrm{i}} /\left(\mathrm{N}_{1}{ }^{2}+\mathrm{N}_{2}{ }^{2}+\mathrm{N}_{3}{ }^{2}\right)^{0.5}$ to calculate the angle $\theta_{i}$ between the detector axes and the burst direction from the values $N_{i}$ of events, where I is the detector number. $\mathrm{N}_{\mathrm{i}}$ values are measured in an experiment for a double time interval of the trigger condition test as the difference in the detected number of gamma quanta and the expected number of background events.

Obviously, the burst source position accuracy depends on the GRB fluence and background level at the trigger time. Computer simulations showed that the accuracy of GRB localisation is up to $2^{\circ}$ for bright bursts with the fluence of $\geq 10^{-5} \mathrm{erg} \mathrm{cm}^{-2}$ measured on the equator and $\sim 5^{\circ}$ for GRBs with a fluence of $\geq 10^{-7} \mathrm{erg} \mathrm{cm}^{-2}$.

\subsection{Optical Observations}

The Lomonosov spacecraft contains two identical devices, namely, SHOK-1 and SHOK2 , each of which is a fast ultra-wide-field camera in the form of a monoblock consisting of an optical photon detection unit, an electronics unit, elements of a mechanical structure and a housing [26].

The SHOK optical unit consists of a recording camera with an optical lens that is mounted on it. The linear dimensions of the camera are $110 \times 66 \times 66 \mathrm{~mm}$, the lens is $\varnothing 69 \times 48 \mathrm{~mm}$. The camera is equipped with a Kodak Truesense KAI-11002 CCD with a resolution of $4008 \times 2672$ pixels and a pixel size of $9 \mu \mathrm{m}$. The camera operates and records optical radiation with a maximum quantum efficiency of $50 \%$ at a wavelength of $500 \mathrm{~nm}$. The camera allows for recording and reading images at a rate of five frames per second at maximum resolution. Reading occurs without time gaps between images.

The lens is attached to the camera with a special flange. The lens has a focal length of $50 \mathrm{~mm}$ and a relative aperture of $\mathrm{f} / \mathrm{d}=1.2$. With these parameters, in combination with the camera used, a field of view of 1000 square degrees is provided. In this case, the average image quality (full width at half maximum (FWHM)) over the field of view is FWHM $\sim 3$ pixels. Taking into account the image quality, camera quantum efficiency, noise, etc., the optical unit has a sensitivity that allows for the detection of optical radiation that corresponds to 9-10 magnitudes in a single frame in the shooting mode with the maximum possible frequency (five frames per second), and when 100 frames are summed up, the sensitivity reaches 13 magnitudes. 
The SHOK FOV is located in the area of GRB detection using the BDRG instrument (see Figure 1). In fact, cameras continuously make a "film", part of which can be transmitted to Earth when a GRB is detected. The cameras are rigidly fixed on the spacecraft platform and rotate with it. Thus, the entire sky is scanned. Image processing is possible between GRB detections to search for optical transients. For this, differential processing of frames is carried out on board in real time. As a result, all moving objects (close asteroids, satellites, space debris), strongly brightened objects and uncatalogued objects remain in the difference frame. Data on all discovered new objects and images of the brightest of them are transmitted to the Earth during the day for further analysis.

In the search mode for optical transients, the camera continuously shoots the sky with low exposures ( $0.2 \mathrm{~s}$ by default). The frames are transferred to the electronic unit for processing, compression and storage. Processing is carried out according to the difference scheme, i.e., the previous one is subtracted from the received frame, and new objects are searched for in the difference. When a bright object is detected, information about it is saved to a special file. Once an hour, for 100 of the brightest events detected during a given hour from the initial set of full-frame images of frames, cutouts centred on the detected object are generated in a time interval of the order of several minutes before and after the event. After their formation, these clippings are saved separately for transmission to Earth. Since the size of the cropped frames is small, this allows for a fairly large number of bright transients to be transmitted to Earth. In the basic mode of operation, the processing program finds all bright, rapidly changing objects.

Due to the large amount of data that is accumulated during film recording, there are two modes of data transmission. The first is the so-called trigger mode, which provides for the transmission of a film recording over the whole camera FOV a few dozens of seconds before and after a BDRG trigger. The second mode is continuous fixation, and then the transmission of data frames is achieved as described above.

The Lomonosov spacecraft became the first Russian satellite with the Internet on board. This made it possible to issue target designations (alerts) from the most striking and interesting events in real time to the ground-based observatories for a more detailed study.

The SHOK optical cameras on board the Lomonosov satellite are completely identical to those used in the MASTER ground network, which includes both wide-field optical cameras and robotic telescopes, which are capable of being quickly redirected to the localisation area of a transient source on the sky [32].

The global network of robotic telescopes MASTER (see Figure 2) is simultaneously (1) a network of alert telescopes that instantly reacts to target designations (alerts) coming from the Gamma-ray Coordinates Network (GCN) or directly via a socket from LIGO/Virgo, IceCube and ANTARES, allowing for detecting optical photons from sources of various physical nature, and (2) a network of robotic telescopes that independently surveys the sky to search for optical transients and issue target designations to other observatories.

The network of robotic telescopes MASTER (see Figure 2) includes eight observatories: MASTER-Amur, MASTER-Tunka, MASTER-Ural, MASTER-Kislovodsk, MASTER-Tavrida (located in Russia), MASTER-SAAO (South Africa), MASTER-IAC (Spain) and MASTEROAFA (Argentina) [32]. Each observatory in the network is capable of surveying the sky at a speed of up to $128 \mathrm{sq}$. deg. per hour and the limiting stellar magnitude on a moonless night is about $20 \mathrm{~m}$. Each observatory is equipped with two spike-angle telescopes with a total field of view of 8 sq. deg. when using a $4098 \times 4098$ pix CCD camera with an image scale of $1.85^{\prime \prime}$ / pix, as well as a photometer with Johnson's BVRI filters and polarisers $[33,34]$.

All alerts from the Lomonosov spacecraft are transmitted to the ground via the Globalstar channels with a minimum time delay, and any telescope in the world will be able to observe the GRB detected by the Lomonosov spacecraft. However, regardless of this, observations by the MASTER network are very important for several reasons. First, observing GRBs, including those from the Lomonosov spacecraft, is a priority task for the MASTER network $[35,36]$. Second, most of the GRBs that are detected on the Lomonosov spacecraft are recorded by the BDRG instrument and have rather wide error areas (1 sq. deg. or 
more). Therefore, the primary task of optical observations, i.e., the detection of an optical companion of a GRB was not possible with conventional narrow-field and/or non-robotic telescopes.

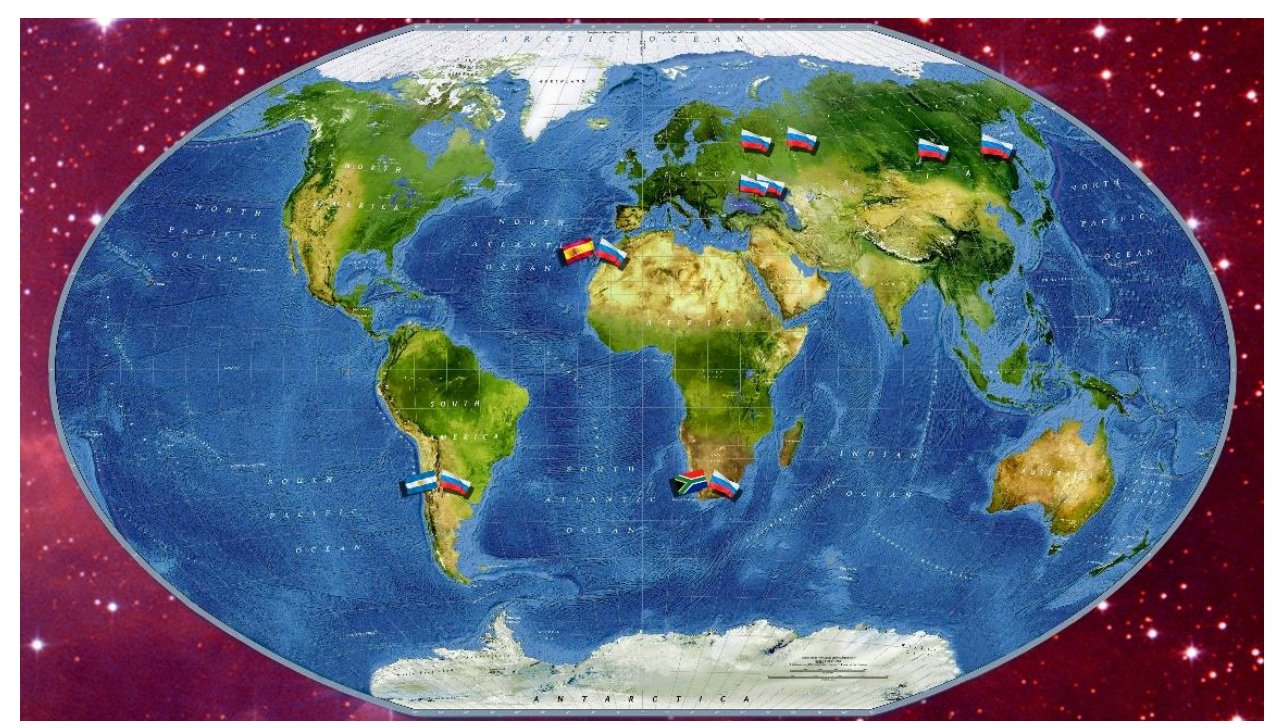

Figure 2. A sketch of the global MASTER robotic network for space monitoring. The facility locations of the corresponding participants are marked by flags on the map.

\section{Results of GRB Observations}

\subsection{Expected Number of Detected GRBs}

To estimate the approximate number of gamma-ray bursts that will be detected in the Lomonosov/BDRG experiment, it is necessary to take into account both the detector parameters and the background conditions in orbit. The measured count rates in the 25-100 keV NaI(Tl) channel of $\sim 2 \times 10^{2} \mathrm{~s}^{-1}$ near the equator and $\sim 10^{3} \mathrm{~s}^{-1}$ at the polar caps were used. Then, we took the spectrum of a typical gamma-ray burst (optically thin plasma, $\mathrm{kT}=100 \mathrm{keV}$ ) and calculated that bursts with a minimum fluence $\mathrm{S} \sim 10^{-7} \mathrm{erg} / \mathrm{cm}^{2}$ could be detected at the equator and $S \sim 5 \times 10^{-7} \mathrm{erg} / \mathrm{cm}^{2}$ could be detected at the polar caps. According to the $\log \mathrm{N}-\log \mathrm{S}$ distribution, the number of GRBs with $\mathrm{S}$ not less than $\sim 10^{-7}-10^{-6} \mathrm{erg} / \mathrm{cm}^{2}$ is about 700 per year from all directions $(4 \pi \mathrm{sr})$. Finally, the BDRG field of view is $\sim 2 \pi \mathrm{sr}$, and the real observational time is $\sim 30 \%$ from the total time at the equator and polar regions. Therefore, the number of bursts detected by at least one BDRG unit is $\sim 100$ per year. Localisation of gamma-ray bursts can be achieved when the fields of view of the detectors overlap, i.e., from $\sim \pi / 2 \mathrm{sr}$, which is four times smaller than the FOV of the entire detector. This means that the expected number of well-located events is $\sim 25$ per year.

\subsection{GRB Catalogue}

The experiment on monitoring of gamma-ray bursts on the Lomonosov satellite was carried out from June 2016 to January 2017.

The Lomonosov satellite operated in a polar orbit of $\sim 500 \mathrm{~km}$, passing through the inner and outer radiation belts with an increased and non-constant background in gammaradiation detectors. GRBs are preferably detected in equatorial and polar regions where the background is lower. However, we had to take into account the burst-like events in the detector caused by the secondary gamma rays that are produced by precipitated electrons, especially in polar zones. To avoid a lot of alerts caused by these electrons, the trigger was set at two levels. The first level threshold was chosen to be quite low but was used only for recording gamma-ray data with the best temporal and spectral resolution. Several dozen triggers of this type, mostly caused by particles, occurred per day. When a second-level trigger, the so-called alert trigger, occurred, in addition to recording detailed information 
about streams and spectra, data from the SHOK cameras were recorded and a telegram was sent to Earth via the GlobalStar modem. This type of trigger has a much higher threshold and some additional conditions, such as a suitable geographic location of the satellite.

Two methods were used to search for gamma-ray bursts. The first one was BDRG trigger data analysis. After eliminating particle-induced simulations, the remaining candidates were compared with GCN notes, as well as with GOES data on solar activity. Several GRBs were confirmed and several outbursts of SGR $1935+2154$ were also found. All BDRG triggers concerning astrophysical GRBs were connected with the events that were also observed in the other space missions. Another method was to search for a response in the monitoring data regarding bursts that were listed in the GCN. Several non-trigger events were added to the Lomonosov GRB catalog after applying this method. Most of them were during the first months of the experiment's flight test when the BDRG trigger algorithm was not optimised.

A catalogue of 20 GRBs observed in the Lomonosov experiment for the period of operation from June 2016 to January 2017 is presented on the site of the mission http: / / lomonosov.sinp.msu.ru/en, accessed on 5 September 2021. All of them are long/soft gamma-ray bursts. The diagrams in Figure 3 present the monthly distribution of detected GRBs and the statistics of the reasons that did not allow us to observe the rest of the GRBs noted in the GCN circulars. The estimated probability of detecting a GRB listed in the GCN is $\sim 20 \%$, taking into account shadowing by the Earth and excluding the radiation belt regions. The probability of detecting a GRB not listed in the GCN is less than 10\% due to the many space missions, such as Swift, Fermi, Wind-Konus and others, which are continuously observing the entire sky. However, among the bursts present in the BDRG catalog, some were not observed by major specialised missions. For example, GRB160908A was presented in GCN only in the CALET experiment [37]. For the burst GRB160915 that was observed by all three BDRG detectors, the GCN has no information on the location of the burst source.

Not a single GRB happened to be in the SHOK cameras' FOVs when this optical instrument was operating. However, three bursts in the BDRG catalogue were observed by telescopes of the MASTER network. In two of them, no optical transient was discovered. Correspondent upper limits were obtained a $16.5 \mathrm{st}$.mag for GRB160720A at $30794 \mathrm{~s}$ after the trigger [38] (the weather conditions were not good for this case) and an 18 st.mag for GRB160824B at 1165 s after trigger [39]. In the cases of GRB161017 and GRB160625B, optical transients were discovered. The results of these observations are presented in detail in the next section.

A list of GRBs detected using BDRG/Lomonosov is presented in Table 3, which also presents the main GRB parameters, i.e., the name containing the registration date; time of the Lomonosov trigger, which corresponds to the time of the burst intensity increasing; duration; energy range where the signal was significant (no less than $5 \sigma$ in at least one detector); and number of Lomonosov's GCN circular and spectral parameters in the power-law exponential cut-off (or briefly cut-off power law (CPL)) approximation, i.e., the following relation for spectral density energy dependence: $J(E)=J_{0} E^{-\gamma} \exp \left(-E / E_{0}\right)$. 


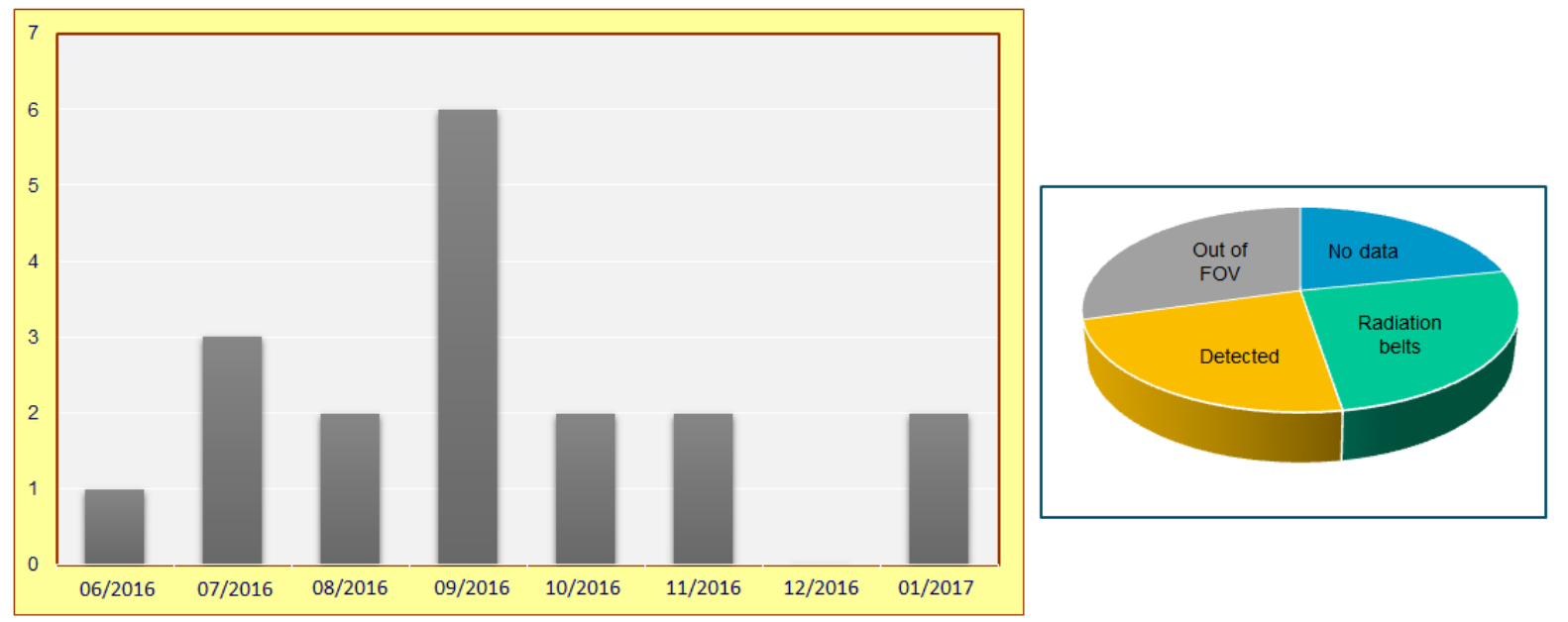

Figure 3. Statistics of GRBs observed by BDRG/Lomonosov.

Table 3. List of GRBs detected using BDRG/Lomonosov.

\begin{tabular}{|c|c|c|c|c|c|c|c|c|}
\hline \multirow{2}{*}{ Event \# } & \multirow{2}{*}{ GRB Name } & \multirow{2}{*}{$\begin{array}{c}\text { Lomonosov } \\
\text { Trigger Time, } \\
\text { UTC }\end{array}$} & \multirow{2}{*}{ Duration, s } & \multirow{2}{*}{$\begin{array}{l}\text { Range, } \\
\mathrm{MeV}\end{array}$} & \multirow{2}{*}{$\begin{array}{l}\text { Lomonosov } \\
\text { GCN Circular }\end{array}$} & \multicolumn{2}{|c|}{$\begin{array}{l}\text { CPL Model } \\
\text { Parameters }\end{array}$} & \multirow{2}{*}{ Detection in Other Missions } \\
\hline & & & & & & $\begin{array}{c}\text { Power } \\
\text { Index, } \gamma\end{array}$ & $\mathrm{E}_{0}, \mathrm{keV}$ & \\
\hline 1 & GRB160607A & $11: 13: 56$ & 17 & $0.035-0.17$ & - & & & Swift, K-W, Integral, CALET \\
\hline 2 & GRB160625B & $22: 51: 16$ & $>20$ & $0.035-0.17$ & - & & & $\begin{array}{c}\text { Swift, Fermi, K-W, CALET, Integral, NA } \\
\text { published }\end{array}$ \\
\hline 3 & GRB160703 & 12:10:02 & 42 & $0.01-0.3$ & 19,725 & & & Swift, K-W, Integral, AstroSat \\
\hline 4 & GRB160705 & 17:40:19 & 3 & $0.035-0.3$ & 19,725 & & & K-W \\
\hline 5 & GRB160720A & 18:23:55 & $>87$ & $0.01-0.8$ & 19,728 & 0.6 & 100 & $\begin{array}{c}\text { Fermi, K-W, CALET, Integral, AstroSat, } \\
\text { HEND }\end{array}$ \\
\hline 6 & GRB160802A & $6: 13: 30$ & 20 & $0.02-0.45$ & 19,759 & 0.8 & 140 & $\begin{array}{c}\text { Fermi, K-W, Integral, AstroSat, HEND, } \\
\text { Mong. N/R }\end{array}$ \\
\hline 7 & GRB160814A & $21: 12: 42$ & 25 & $0.02-0.17$ & 21,402 & & & K-W, CALET, MAXI, \\
\hline 8 & GRB160824B & $14: 21: 07$ & 3 & $0.25-1.0$ & 19,884 & & & Fermi, K-W, CALET, Integral, AstroSat \\
\hline 9 & GRB160908A & $5: 17: 06$ & 30 & $0.01-0.3$ & - & & & K-W, CALET, Integral \\
\hline 10 & GRB160911 & $14: 07: 55$ & 20 & $0.1-0.5$ & - & & & K-W \\
\hline 11 & GRB160912 & $8: 24: 09$ & 21 & $0.06-0.9$ & - & & & Fermi, K-W, Integral \\
\hline 12 & GRB160915A & $22: 51: 12$ & 42 & $0.12-1.0$ & 19,989 & -0.5 & 70 & K-W, Integral \\
\hline 13 & GRB160917B & $10: 56: 12$ & 4 & $0.02-0.8$ & 19990 & & & $\begin{array}{c}\text { Fermi, K-W, Integral } \\
\text { Swift, Fermi, Integral, AstroSat, Maid, }\end{array}$ \\
\hline 14 & GRB160917A & $11: 30: 20$ & 30 & $0.035-0.8$ & 19,992 & & & $\begin{array}{l}\text { Mondy, TSHAO, http:// www.mpe.mpg. } \\
\text { de/ jicg/grb160917A.html accessed on } 5 \\
\text { September } 2021\end{array}$ \\
\hline 15 & GRB161015A & 17:03:06 & 15 & $0.13-1.1$ & - & & & Swift, Fermi, K-W, Integral, AstroSat \\
\hline 16 & GRB161017A & $17: 52: 17$ & 100 & $0.02-0.3$ & 20,075 & 0.9 & 230 & Swift, Fermi, K-W \\
\hline 17 & GRB161106A & $11: 57: 55$ & 10 & $0.02-0.8$ & 20,154 & 0.3 & 60 & Fermi, K-W, CALET \\
\hline 18 & GRB161109A & $6: 19: 02$ & 4 & $0.035-0.3$ & 20,165 & & & Swift, Fermi, K-W, CALET \\
\hline 19 & GRB170101A & 2:26:00 & 3 & $0.01-0.8$ & 20,359 & 0.7 & 100 & $\begin{array}{l}\text { Swift, K-W, POLAR, AAO, http:/ / www. } \\
\text { mpe.mpg.de/ jcg/grb170101A.html } \\
\text { accessed on 5 September } 2021\end{array}$ \\
\hline 20 & GRB170101B & 2:47:22 & 17 & $0.035-0.3$ & 20,360 & & & Fermi, AstroSat, POLAR \\
\hline
\end{tabular}

The spectral parameters $\gamma$ and $\mathrm{E}_{0}$ were estimated only for those events for which significant signals were detected in no less than three energy ranges in one of the BDRG detectors. Information about other space missions in which a given GRB was observed is also presented in a separate column.

Light curves for all events, except GRB160625B and GRB161017A, obtained in several energy channels by the best-illuminated BDRG boxes are shown in Figures 4-6. The monitoring data with a time resolution of $0.1 \mathrm{~s}$ were used. These light curves are presented for energy ranges in which the detected signals were more significant. They correspond to the ranges presented in Table 3. The events GRB160625B and GRB161017A are discussed in the next section. Their light curves were presented in $[40,41]$. 


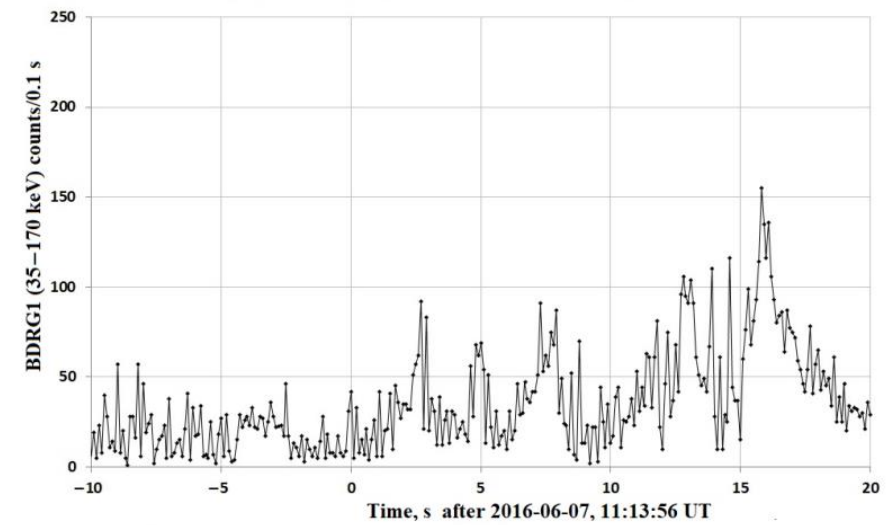

(a)

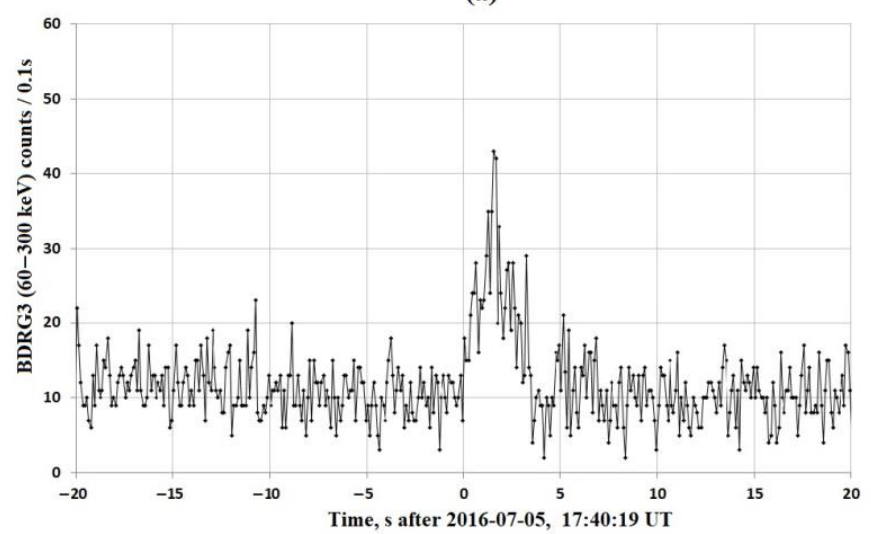

(c)

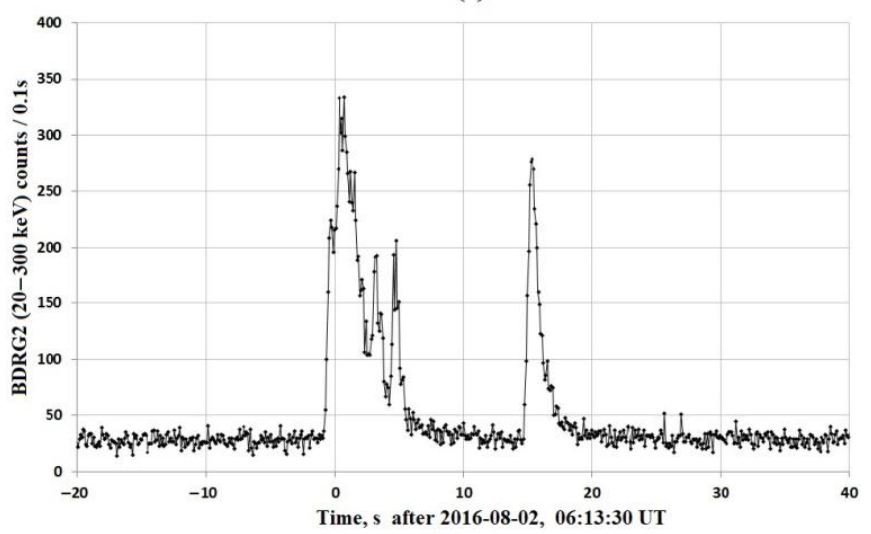

(e)

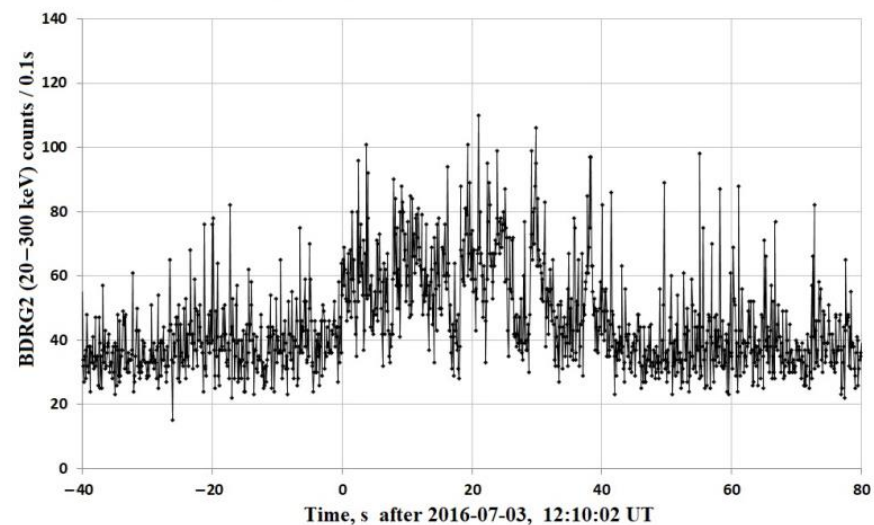

(b)

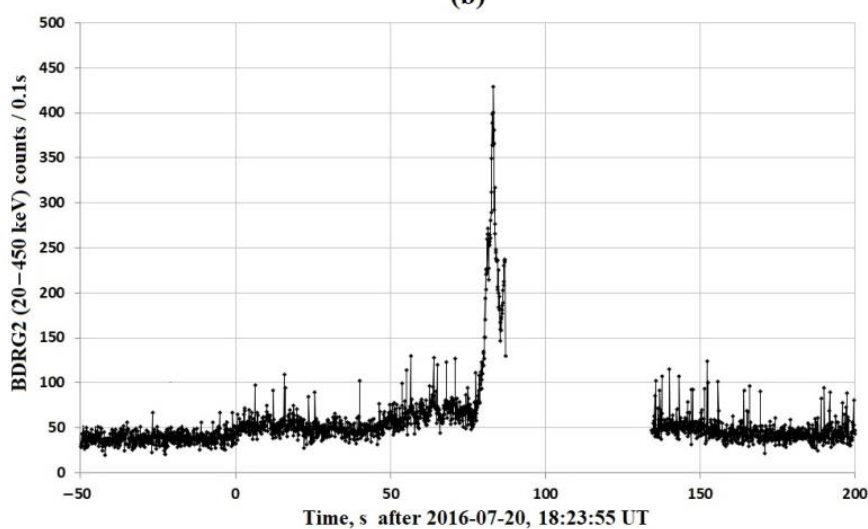

(d)

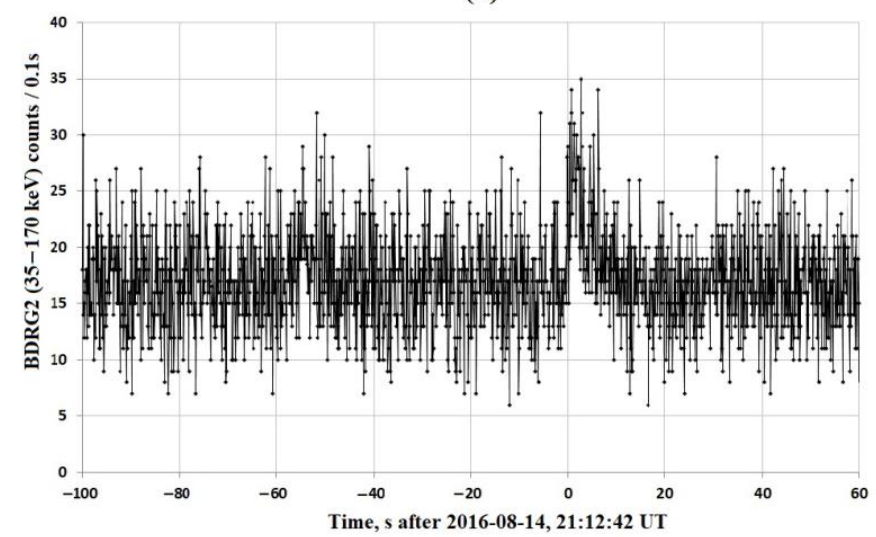

(f)

Figure 4. Light curves of BDRG/Lomonosov GRBs. (a) 2016-06-07, 11:13:56 UT; (b) 2016-07-03, 12:10:02 UT; (c) 2016-07-07, 17:40:19 UT; (d) 2016-07-20, 18:23:55 UT; (e) 2016-08-02, 06:13:30 UT; (f) 2016-08-14, 21:12:42 UT. 


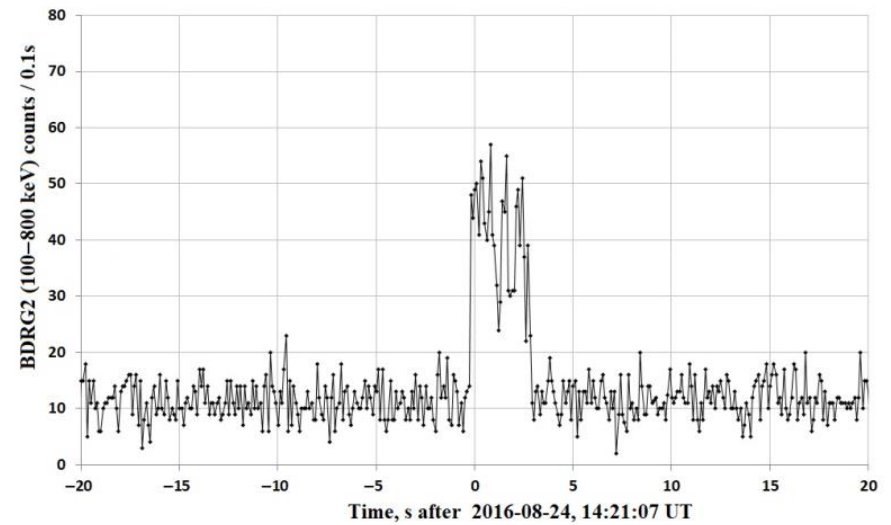

(a)

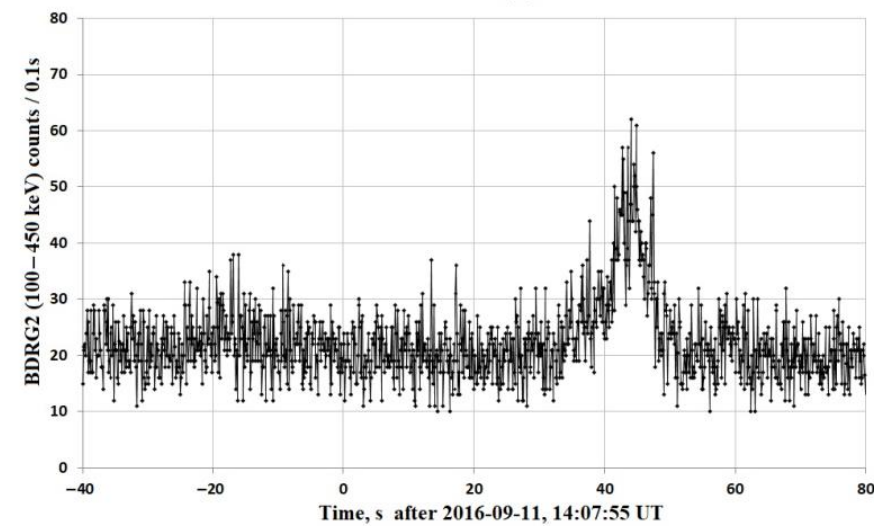

(c)

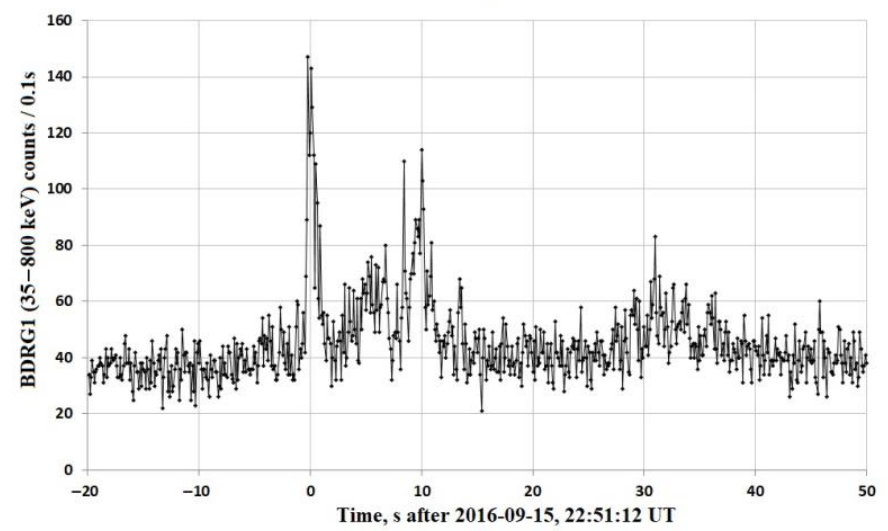

(e)

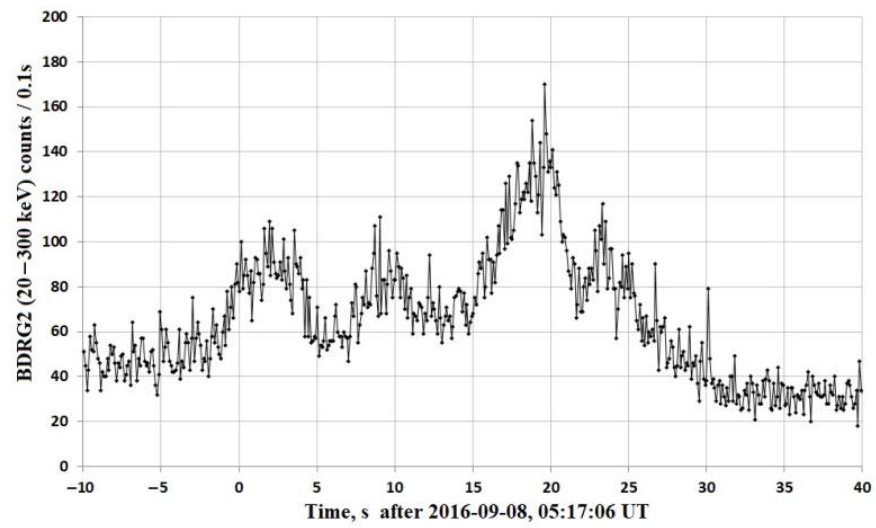

(b)

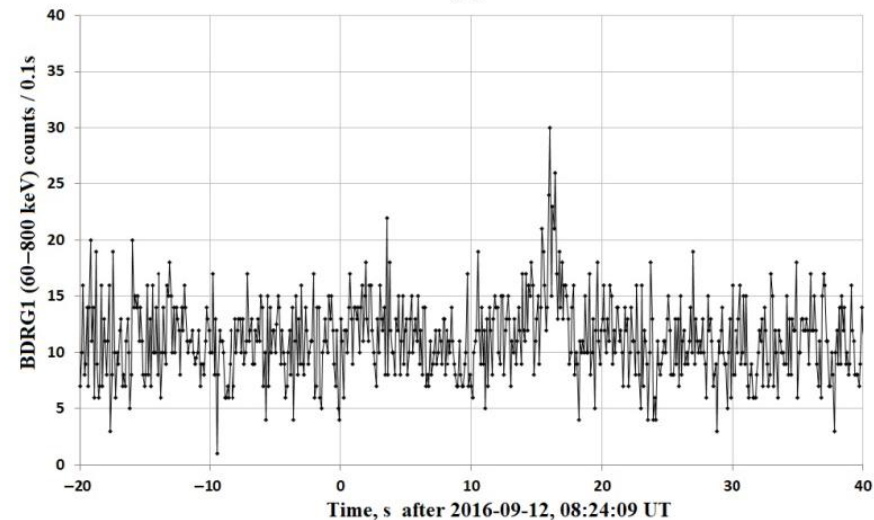

(d)

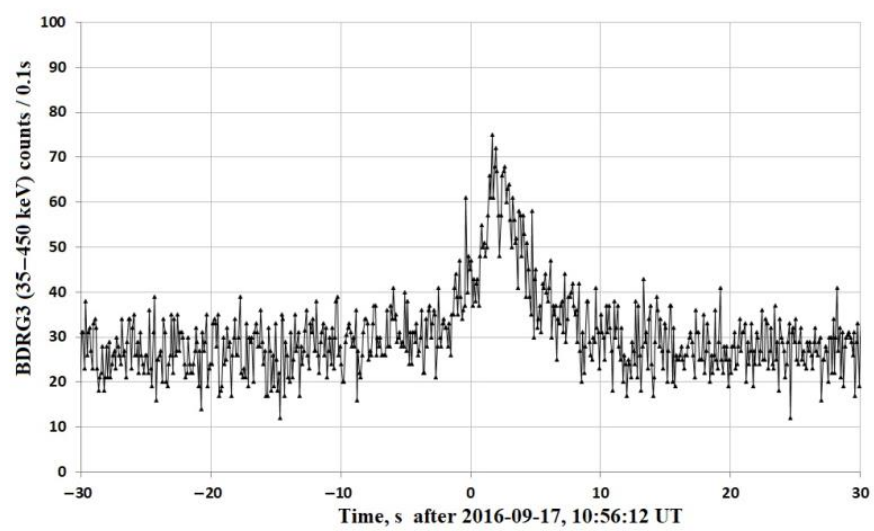

(f)

Figure 5. Light curves of BDRG/Lomonosov GRBs. (a) 2016-08-24, 14:21:07 UT; (b) 2016-09-08, 05:17:06 UT; (c) 2016-09-11, 14:07:55 UT; (d) 2016-09-12, 08:24:09 UT; (e) 2016-09-15, 22:51:12 UT; (f) 2016-09-17, 10:56:12 UT. 


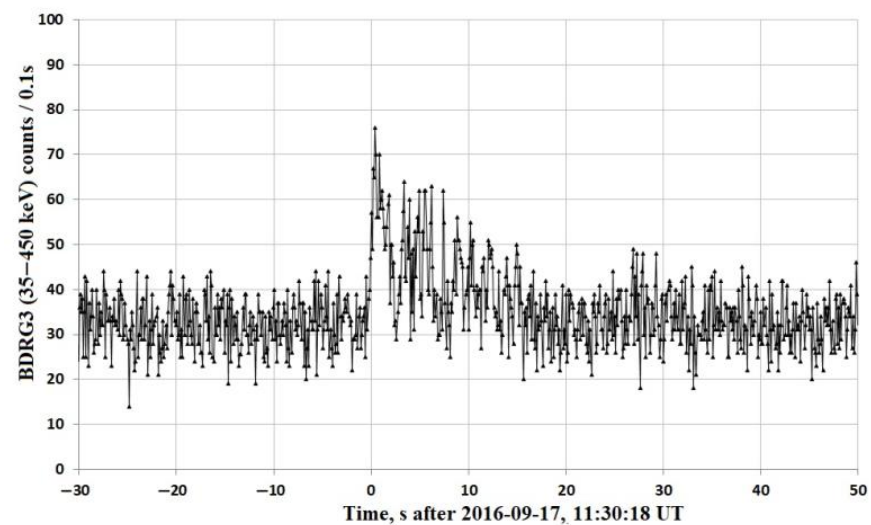

(a)

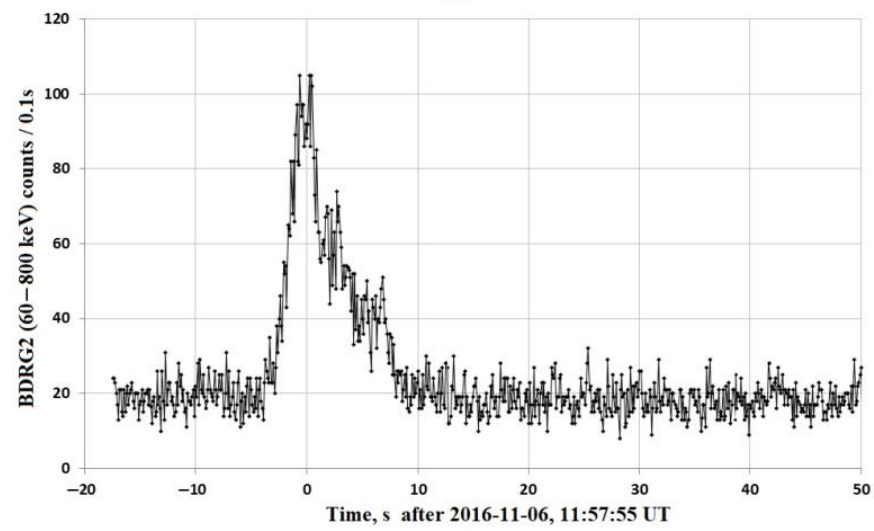

(c)

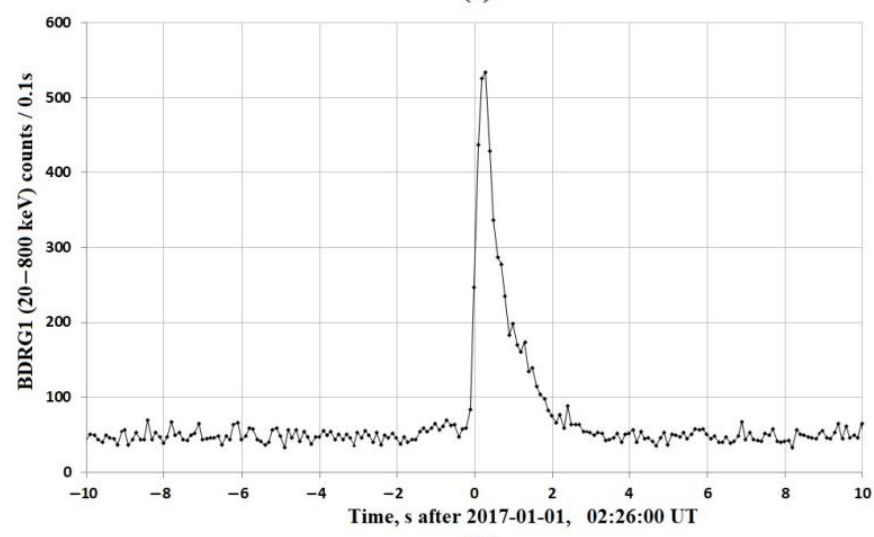

(e)

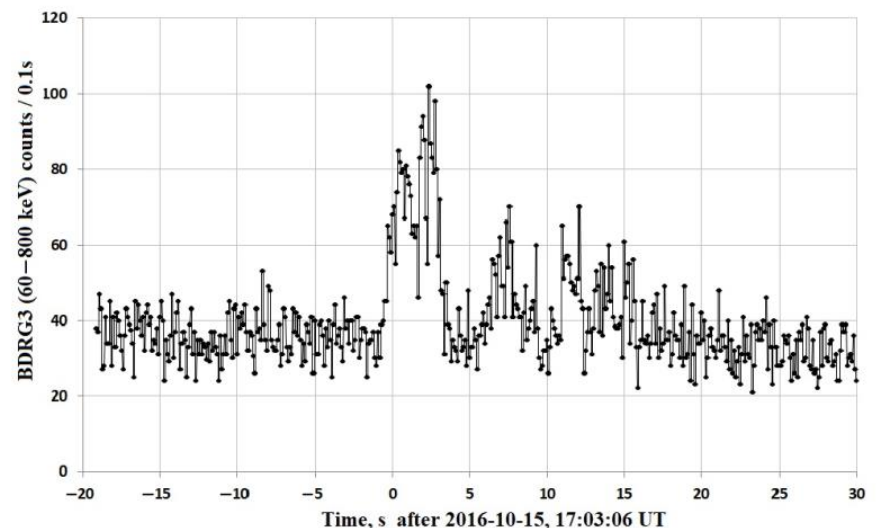

(b)

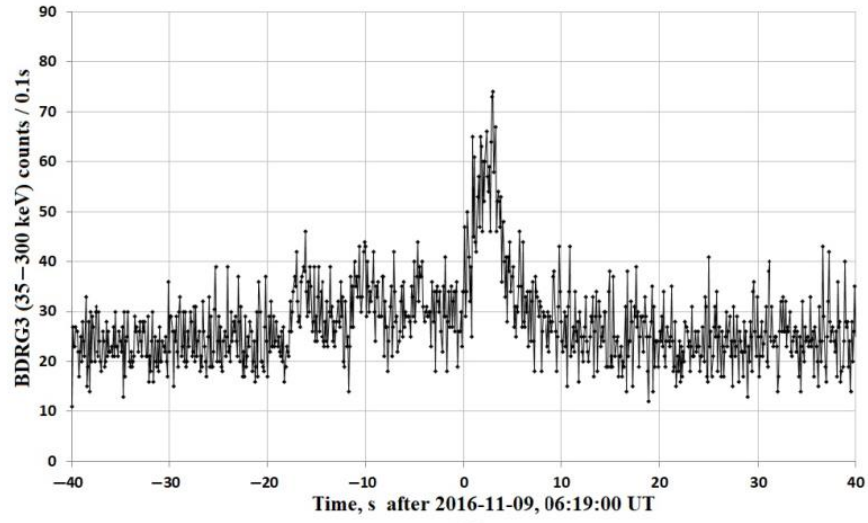

(d)

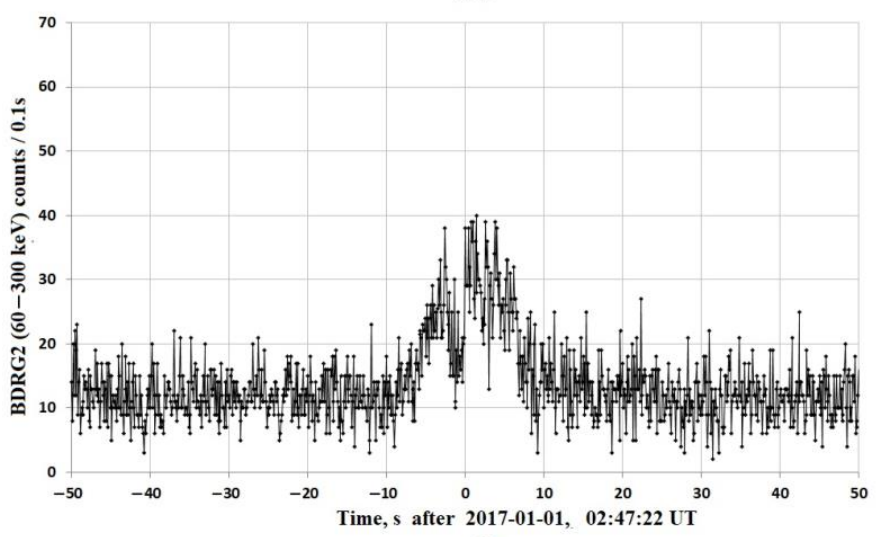

(f)

Figure 6. Light curves of BDRG/Lomonosov GRBs. (a) 2016-09-17, 11:30:18 UT; (b) 2016-10-15, 17:03:06 UT; (c) 2016-11-06, 11:57:55 UT; (d) 2016-11-09, 06:19:00 UT; (e) 2017-01-01, 02:26:00 UT; (f) 2017-01-01, 02:47:22 UT.

\subsection{Multi-Messenger and Multi-Wavelength Observations}

Among the GRBs that were detected on Lomonosov by the BDRG instrument, two events, i.e., GRB161017A and GRB160625B, were also observed by ground telescopes in different wavebands.

GRB160625B is the most powerful recorded gamma-ray burst. It was detected on 25 June 2016 at 22:40:16UT by the Enrico Fermi Space Observatory (NASA, Washington, D.C., USA, [42]), first as a short pulse (Fermi-GBM trigger 488587220, [43]). At 22:43:24.82, the Fermi-LAT triggered on a bright pulse from the same GRB [44]. At 22:51:16.03, the Fermi-GBM triggered again (trigger 488587880). The GBM light curve consisted of multiple peaks over approximately $700 \mathrm{~s}$, with the first one being a $1 \mathrm{~s}$ long soft peak. The main peak, corresponding to the LAT trigger, was very hard and about $25 \mathrm{~s}$ long. The peak that triggered the GBM for the second time was soft and about $30 \mathrm{~s}$ long [43]. 
Due to its outstanding brightness, GRB160625B was observed by a large number of space and ground telescopes in a wide range of electromagnetic waves from gamma rays to radio waves ([43-50] and others). Many papers were published on this event, including some by the authors of this work ([51-57] and others). Particularly, for the first time in the history of the study of gamma-ray bursts, the polarisation of its optical radiation synchronous with the gamma was detected [45]. GRB160625B also triggered detectors BDRG on board the Lomonosov Space Observatory of Moscow State University [24] and Konus-Wind at 22:40:19.875UT [49], as well as the CALET Gamma-Ray Burst Monitor (CGBM) at 22:40:15.49 [58]. Swift-XRT detected an uncatalogued X-ray source at the position with R.A.(J2000) $=$ 20:34:23.25 and Dec.(J2000) $=+06: 55: 10.5$ [47], which is likely the X-ray counterpart of GRB160625B.

The optical counterpart was discovered by RATIR [59] starting $8.53 \mathrm{~h}$ after the LAT trigger. The detection of a significant $(8.3 \pm 0.8 \%$ from our most conservative estimation) variable linear polarisation of the prompt optical flash that accompanied the extremely energetic and long prompt $\gamma$-ray emission from GRB160625B was discovered by MASTER $[45,51,60]$. MASTER started its observation using polarisation filters $[32,33,61] 31 \mathrm{~s}$ after the GBM notice time (57 s after GBM, i.e., $131 \mathrm{~s}$ before the LAT trigger [44]) at 201606-25 22:41:13UT [60], but published the circular in the GCN [62,63] at 16/06/28 14:05:38. The GCN publication was delayed by the installation of the new MASTER telescope in Argentina (MASTER-OAFA).

On 25 June 2016, near the GRB160625B trigger, the BDRG/Lomonosov operated in monitoring mode. The background environment at the time of the event was very complicated because the Lomonosov satellite was flying through radiation belts. The presented light curves clearly point to a significant increase of hard X-ray and soft gammaray fluxes near $650 \mathrm{~s}$ after the BAT/Swift trigger or about $450 \mathrm{~s}$ after the GBM/Fermi trigger, which is evidence of the central engine operation $10 \mathrm{~min}$ after the main explosion. The light curve recorded by the BDRG/Lomonosov instrument with a time resolution of $0.1 \mathrm{~s}$ demonstrated a fine structure as several pulses, with the most intensive at 22:51:16UT and less intense and wider at about $100 \mathrm{~s}$ beforehand. This time structure was confirmed by the GBM/Fermi data. The intensive pulse at 22:51:16UTC, as well as preceding observations, were clearly in the energy range from 3.4 to $44 \mathrm{keV}$, while from the BDRG/Lomonosov data, they could be observed in an energy interval from $25 \mathrm{keV}$ up to $170 \mathrm{keV}$. The event was observed approximately $140 \mathrm{~s}$ after the first peak of the burst, which means that the central engine of the GRB continued its operation for quite a long time after the burst inception. The fine structure that was observed on the GRB light curve at dozens of seconds after the beginning of the central engine operation may have been caused by collisions of relativistic shells that propagated in the beam with different Lorentz factors. A more detailed analysis of the multi-wavelength observations by different space and ground instruments, together with the BDRG/Lomonosov observations, was done by Lipunov et al. [40].

Simultaneous observations of the gamma and optical spectra gave unique information about the GRB source, in particular, about the relativistic jet formation in the central engine. MASTER measurements probed the structure of the magnetic field at an early development stage of the jet, closer to a central black hole, and showed that the prompt emission phase was created by rapidly cooling synchrotron radiation in a large-scale magnetic field that was transferred from the black hole and distorted by dissipation processes inside the jet [51]. The optical data obtained by MASTER telescope robots $[32,61]$ had the best temporal resolution with a minimum exposure time of $5 \mathrm{~s}$. This resolution made it possible to suspect quasiperiodic variability in the optical range, which could be associated with the dual nature of the long GRB. The detailed photometry of the gamma-ray burst optical counterpart indicated the presence of quasiperiodic emission components in the intrinsic optical emission of GRB160625B. As a result of synchronous multi-wavelength observations, a three-stage collapse scenario for this long and bright burst could be proposed. The quasiperiodic fluctuations may be associated with a forced precession of a self-gravitating, rapidly rotating superdense body, a so-called spinar, whose evolution is determined by 
a powerful magnetic field. The spinar's mass is such that at the end of its evolution, it collapses into a black hole.

The other GRB for which the multi-wavelength observations were realised was GRB161017A. The data in the gamma range were obtained from BDRG/Lomonosov. The burst was detected in the $20-100 \mathrm{keV}$ channels of $\mathrm{NaI}(\mathrm{Tl})$ detectors at significant levels $4.4 \sigma$ (BDRG-1), $14.1 \sigma$ (BDRG-2) and 0.045 $\sigma$ (BDRG-3). Data in the trigger mode were recorded in the time interval $(-30 s ;+119 s)$ relative to the trigger time. The GRB161017A light curve consisted of at least several periods of gamma emission activity. It indicated the working of a central engine just more than $100 \mathrm{~s}$ after the beginning of the GRB.

GRB161017A was also detected by Swift/BAT [48], which determined the accurate source location. Then, a MASTER robot located near Blagoveshchensk (see Figure 3) automatically pointed there and discovered that an optical transient [64] appeared at $\sim 70 \mathrm{~s}$ from the Swift trigger that was produced at the front of the first peak of the light curve i.e., about $30 \mathrm{~s}$ before the BDRG/Lomonosov GRB trigger. Therefore, the optical emission corresponded to the period of the presence of prompt gamma radiation.

Comparative analysis of the light curves showed that the optical emission was uncorrelated with the $\mathrm{X}$-rays and gamma rays, which had a similar structure. Detailed analysis of GRB161017 was done in [41].

It is necessary to note that in some cases (e.g., [29,65]), GRB optical emissions may correlate with the prompt gamma-ray emission, whereas in other cases, the early optical light curve and the gamma-ray light curve are not correlated. The optical light curve has a smooth structure with a fairly shallow maximum (in logarithmic coordinates) that may correspond to the smooth optical self-similar emission model (SOSSE) proposed by [66]. Because GRB161017A was an extremely long event, the SOSSE mechanism maybe took place in combination with various non-adiabatic effects in the jet, as well as the extended operation of the central engine.

Another example of multi-messenger observations is the BDRG/Lomonosov gammaray prompt observation of the neutrino event IceCube-160806 [67], which was detected at the IceCube neutrino observatory 2016, August, 06 [68]. The center of the error box of this event was in the BDRG FOV, $47^{\circ}$ from the axis of the most illuminated BDRG-1 detector (direction of BDRG-1 axis $\alpha=5$ h 5 min $8.96 \mathrm{~s}, \delta=-25^{\circ} 24^{\prime} 44.54^{\prime \prime}$ ). No significant GRB was detected by the BDRG/Lomonosov in the interval $\pm 50 \mathrm{~s}$ from the moment of IC-160806 (06.08.2016, 12:21:33 UTC). Upper limits ( $3 \sigma)$ for the $1 \mathrm{~s}$ exposition from BDRG-1 background counts was $<0.23 \mathrm{~cm}^{-2} \mathrm{~s}^{-1}$ for $20-60 \mathrm{keV},<0.13 \mathrm{~cm}^{-2} \mathrm{~s}^{-1}$ for $60-300 \mathrm{keV}$.

\subsection{Fine Structure of the GRB170101A Light Curve}

One of the BDRG/Lomonosov experimental advantages is the possibility of eventby-event recording. This gives a good opportunity for studying of GRB light curve fine structure. The time resolution is limited only by the statistics of detected quanta. Examples of the light curves recorded with a high temporal resolution for GRB170101A are presented in Figure 7. When comparing these light curves with the one presented in Figure 6e, some temporal peculiarities are revealed. As it can be seen from Figure 6e, where the light curve recorded with a $100 \mathrm{~ms}$ resolution is presented, its shape is quite typical for a short burst, i.e., a fast rise and slow decay (FRSD) without any pronounced peculiarities. Meanwhile, light curves recorded with a $10 \mathrm{~ms}$ resolution, which are presented in Figure 7, demonstrate (aside from the main maximum at $\sim 0.35 \mathrm{~s}$ ) some structures such as quasiperiodic local maxima (at $\sim 0.2, \sim 0.5, \sim 0.6, \sim 0.75$ and $\sim 1.25 \mathrm{~s}$ ). These maxima were traced independently for all light curves recorded by different BDRG detectors. This indicates that these variations were not caused by statistical fluctuations, but are highly likely to be real. 


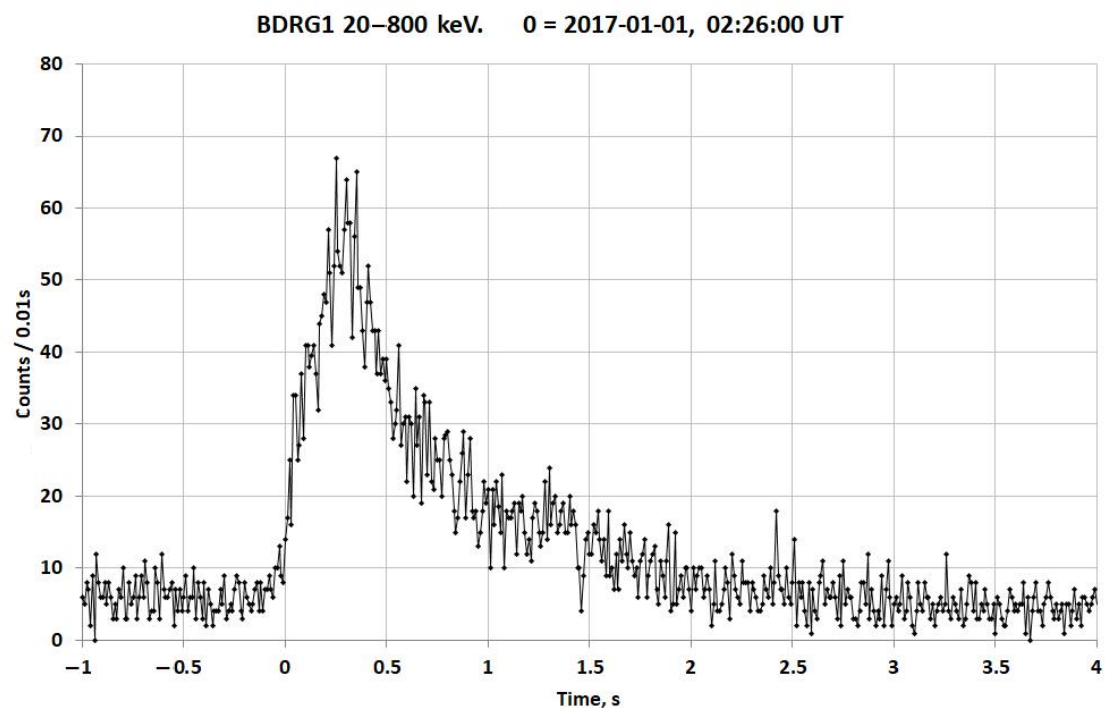

(a)

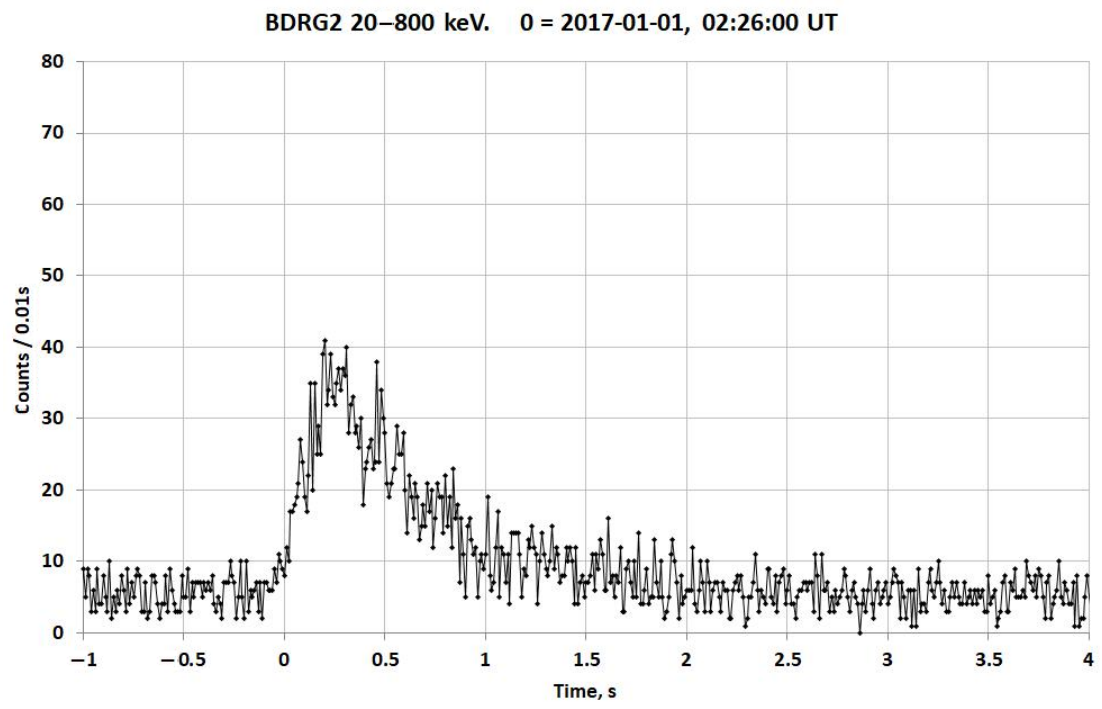

(b)

BDRG3 20-800 keV. $0=$ 2017-01-01, 02:26:00 UT

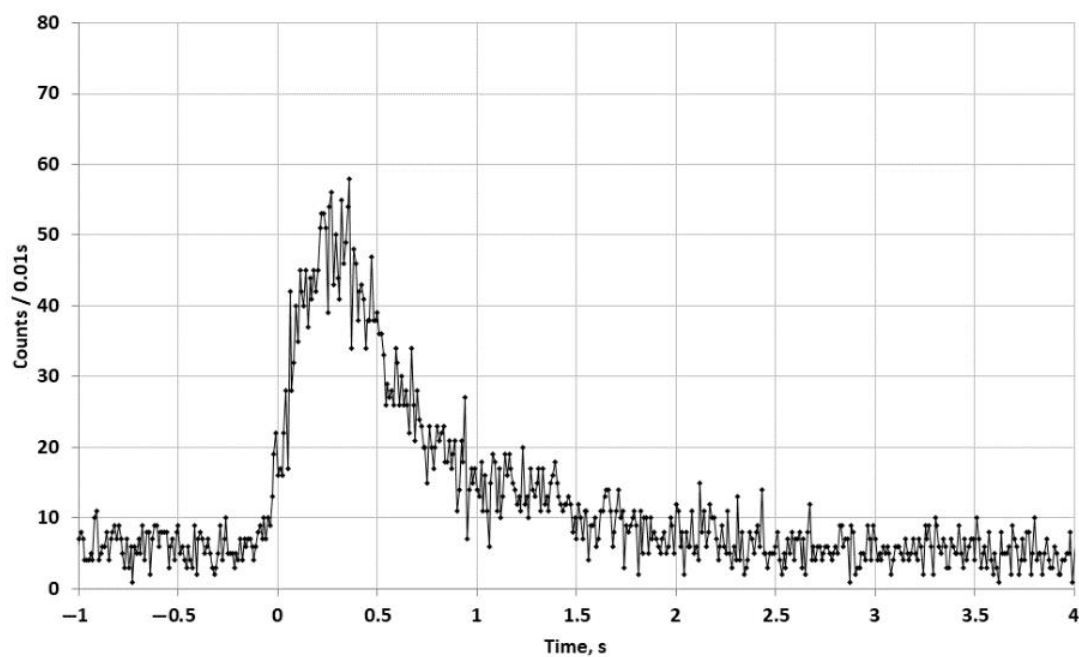

(c)

Figure 7. GRB170101A light curves in the range of the best significance detected by BDRG -1 (a), BDRG -2 (b) and BDRG -3 (c) with a $10 \mathrm{~ms}$ time resolution. 
Such quasi-periodic structures may indicate the spinar character of this GRB source, as in the case of GRB160625B discussed above, for which quasi-periodic variations were revealed in the optical light curve. The other explanation may be connected with quasiregular collisions of shocks that were generated by the central engine, which propagate with different Lorenz factors. In any case, this example demonstrates the necessity and importance of GRB light curve fine structure recording.

\section{Conclusions}

The Lomonosov mission was the first space observatory in which multi-messenger and multi-wavelength observations of GRBs, i.e., the most powerful known explosive events in the Universe, were realised. To carry out these studies, scientific instruments of the X-ray and gamma-ray detector unit (BDRG) and wide-field optical cameras (SHOKs) were used. The scientific payload on the Lomonosov spacecraft was directly connected to the Internet for the first time in the practice of Russian space instrumentation, and through it, the output data flew into the international gamma-ray burst registration network GCN. Synchronous observations of the Universe by detectors located on several scientific spacecraft and ground-based telescopes led to the acquisition of multi-messenger and multi-wavelength data for several gamma-ray bursts. In particular, the joint observations of the Lomonosov GRB monitor with a MASTER net of robotic telescopes allowed for comparing optical and gamma-ray light curves of two long-duration and the most powerful GRBs, i.e., GRB160625B and GRB161017A, at different phases of their activity. These observations made it possible to clarify the details of the birth of rapidly rotating black holes of stellar mass.

It is also necessary to note a significant advantage of the BDRG instrument, namely, the event-by-event recording. This mode made it possible to study fine structures of GRB light curves of hard X-rays and gamma rays.

Author Contributions: Conceptualization, V.A.S., M.I.P., S.I.S., V.M.L. and I.H.P.; methodology, S.I.S.,V.M.L.,V.V.B., A.F.I., V.G.K., V.L.P., N.N.V. and I.V.Y.; software, V.V.B., A.V.B., E.S.G., V.V.K.; validation, S.I.S., V.V.B., V.L.P. and I.V.Y.; formal analysis, V.V.B., A.V.B. and E.S.G.; investigation, S.I.S., V.M.L., V.V.B., A.V.B., E.S.G. and A.F.I.; data curation, V.V.K.; writing-original draft preparation, S.I.S. and V.M.L.; writing - review and editing, S.I.S., A.V.B., A.F.I. and I.H.P.; visualization, A.V.B., V.V.K.; supervision, V.A.S. and M.I.P.; project administration, S.I.S. and I.V.Y. All authors have read and agreed to the published version of the manuscript.

Funding: This research was supported by the Interdisciplinary Scientific and Educational School of Moscow University "Fundamental and Applied Space Research". The BDRG experiment onboard the Lomonosov satellite was realised with the partial financial support of the M.V. Lomonosov Moscow State University within the framework of the "Prospects for Development" program ("Perspektivnye Napravleniya Razvitiya"). The MASTER Global Robotic Net is supported in part by the Development Program of Lomonosov Moscow State University. I.H.P. and N.N.V. acknowledge the support from the National Research Foundation of Korea (NRF-2017K1A4A3015188, NRF-2021R1A2B5B03002645 and NRF-2019H1D3A2A02060090).

Conflicts of Interest: The authors declare no conflict of interest.

\section{References}

1. Salvaterra, R.; Della Valle, M.; Campana, S.; Chincarini, G.; Covino, S.; D'Avanzo, P.; Fernández-Soto, A.; Guidorzi, C.; Mannucci, F.; Margutti, R.; et al. GRB 090423 at a redshift of $\mathrm{z} \approx 8.1$. Nature 2009, 461, 1258-1260. [CrossRef]

2. Zhang, B.; Zhang, B.-B.; Virgili, F.J.; Liang, E.-W.; Kann, D.A.; Wu, X.-F.; Proga, D.; Lv, H.-J.; Toma, K.; Mészáros, P.; et al. Discerning the Physical Origins of Cosmological Gamma-Ray Bursts Based on Multiple Observational Criteria: The Cases of $\mathrm{z}=6.7$ GRB 080913, z = 8.2 GRB 090423, and Some Short/Hard GRBs. ApJ 2009, 703, 1696-1724. [CrossRef]

3. Lipunov, V.M.; Postnov, K.A.; Prokhorov, M.E. Gamma-ray bursts as standard-energy explosions. Astron. Rep. 2001, 45, 236-240. [CrossRef]

4. Amati, L.; Guidorzi, C.; Frontera, F.; Della Valle, M.; Finelli, F.; Landi, R.; Montanari, E. Measuring the cosmological parameters with the Ep,i-Eiso correlation of gamma-ray bursts. MNRAS 2008, 391, 577-584. [CrossRef] 
5. Ghirlanda, G.; Ghisellini, G.; Firmani, C. Gamma ray bursts as standard candles to constrain the cosmological parameters. New J. Phys. 2006, 8, 123.

6. Panaitescu, A.; Vestrand, W. Taxonomy of gamma-ray burst optical light curves: Identification of a salient class of early afterglows. MNRAS 2006, 387, 497-504. [CrossRef]

7. Perlmutter, S.; Aldering, G.; Goldhaber, G.; Knop, R.A.; Nugent, P.; Castro, P.G.; Deustua, S.; Fabbro, S.; Goobar, A.; Groom, D.E.; et al. Measurements of $\Omega$ and $\Lambda$ from 42 High-Redshift Supernovae. ApJ 1999, 51, 565-586. [CrossRef]

8. Perley, D.A.; Bloom, J.S.; Klein, C.R.; Covino, S.; Minezaki, T.; Wozniak, P.; Vestrand, W.T.; Williams, G.G.; Milne, P.; Butler, N.R.; et al. Evidence for supernova-synthesized dust from the rising afterglow of GRB 071025 at z 5. MNRAS 2010, 406, 2473-2487. [CrossRef]

9. Munshi, D.; Valageas, P.; Van Waerbeke, L.; Heavens, A. Cosmology with weak lensing surveys. Phys. Rep. 2008, 462, 67-121. [CrossRef]

10. Greiner, J.; Krühler, T.; Klose, S.; Afonso, P.; Clemens, C.; Filgas, R.; Hartmann, D.H.; Küpcü Yoldaş, A.; Nardini, M.; Olivares, F.E.; et al. The nature of "dark" gamma-ray bursts. Astron. Astrophys. 2011, 526, 10. [CrossRef]

11. Waxman, E. Gamma-ray bursts: Potential sources of ultra high energy cosmic-rays. Nucl. Phys. B Proc. Suppl. 2006, 151, 46-53. [CrossRef]

12. Cutler, C.; Thorne, K.S. An Overview of Gravitational-Wave Sources. arXiv 2002, arXiv:gr-qc/0204090. Available online: https://arxiv.org/abs/gr-qc/0204090 (accessed on 5 September 2021).

13. Halzen, F.; Klein, S.R. Review of Scientific Instruments 81 081101. 2010. Available online: https://arxiv.org/abs/1007.1247 (accessed on 5 September 2021).

14. Gorham, P.W.; Allison, P.; Barwick, S.W.; Beatty, J.J.; Besson, D.Z.; Binns, W.R.; Chen, C.; Chen, P.; Clem, J.M.; Connolly, A.; et al. The Antarctic Impulsive Transient Antenna Ultra-high Energy Neutrino Detector Design, Performance, and Sensitivity for 2006-2007 Balloon Flight. Astropart. Phys. 2009, 32, 10-41. [CrossRef]

15. Abramovici, A.; Althouse, W.E.; Drever, R.W.P.; Gursel, Y.; Kawamura, S.; Raab, F.J.; Shoemaker, D.; Sievers, L.; Spero, R.E.; Thorne, K.S.; et al. LIGO: The Laser Interferometer Gravitational-Wave Observatory. Science 1992, 256, 325-333. [CrossRef]

16. Pau, A.-S.; Aoudia, S.; Babak, S.; Binétruy, P.; Berti, E.; Bohé, A.; Caprini, C.; Colpi, M.; Cornish, N.J.; Danzmann, K.; et al. eLISA: Astrophysics and cosmology in the millihertz regime. arXiv 2012, arXiv:1201.3621. Available online: https://arxiv.org/abs/1201 .3621 (accessed on 5 September 2021).

17. Abu-Zayyad, T.; Aida, R.; Allen, M.; Anderson, R.; Azuma, R.; Barcikowski, E.; Belz, J.W.; Bergman, D.R.; Blake, S.A.; Cady, R.; et al. The surface detector array of the Telescope Array 2 experiment. Nucl. Instr. Meth. A 2012, 689, 87-97. [CrossRef]

18. Tokuno, H.; Tameda, Y.; Takeda, M.; Kadota, K.; Ikeda, D.; Chikawa, M.; Fujii, T.; Fukushima, M.; Honda, K.; Inoue, N.; et al. New air fluorescence detectors employed in the Telescope Array experiment. Nucl. Instr. Meth. A 2012, 676, 54-65. [CrossRef]

19. Abraham, J.; Aglietta, M.; Aguirre, I.C.; Albrow, M.G.; Allard, D.; Allekotte, I.; Allison, P.; Alvarez-Muniz, J.; do Amaral, M.G.; Ambrosio, M.; et al. Properties and performance of the prototype instrument for the Pierre Auger Observatory. Nucl. Instr. Meth. A 2004, 523, 50-95. [CrossRef]

20. Panasyuk, M.I.; Casolino, M.; Garipov, G.K.; Ebisuzaki, T.; Gorodetzky, P.; Khrenov, B.A.; Klimov, P.A.; Morozenko, V.S.; Sakaki, N.; Saprykin, O.A.; et al. The current status of orbital experiments for UHECR studies. J. Phys. Conf. Ser. 2015, 632, 012097. [CrossRef]

21. Takahashi, Y. and the JEM-EUSO Collaboration. The JEM-EUSO mission. New J. Phys. 2009, 11, 065009. Available online: https:/ /iopscience.iop.org/article/10.1088/1367-2630/11/6/065009 (accessed on 5 September 2021). [CrossRef]

22. Costa, E.; Frontera, F.; Heise, J.; Feroci, M.; in 't Zand, J.; Fiore, F.; Cinti, M.N.; Dal Fiume, D.; Nicastro, L.; Orlandini, M.; et al. Discovery of an X-ray afterglow associated with the $\gamma$-ray burst of 28 February 1997. Nature 1997, 387, 783-785. [CrossRef]

23. Park, I.H.; Brandt, S.; Budtz-Jørgensen, C.; Castro-Tirado, A.J.; Chen, P.; Connell, P.; Eyles, C.; Grossan, B.; Huang, M.-H.A.; Jeong, S.; et al. Ultra-Fast Flash Observatory for the observation of early photons from gamma-ray bursts. New J. Phys. 2013, 15, 023031. [CrossRef]

24. Sadovnichii, V.A.; Panasyuk, M.I.; Amelyushkin, A.M.; Bogomolov, V.V.; Benghin, V.V.; Garipov, G.K.; Kalegaev, V.V.; Klimov, P.A.; Khrenov, B.A.; Petrov, V.L.; et al. "Lomonosov" Satellite-Space Observatory to Study Extreme Phenomena in Space. Space Sci. Rev. 2017, 212, 1705-1738. [CrossRef]

25. Svertilov, S.I.; Panasyuk, M.I.; Bogomolov, V.V.; Amelushkin, A.M.; Barinova, V.O.; Galkin, V.I.; Iyudin, A.F.; Kuznetsova, E.A.; Prokhorov, A.V.; Petrov, V.L.; et al. Wide-Field Gamma-Spectrometer BDRG: GRB Monitor On-Board the Lomonosov Mission. Space Sci. Rev. 2018, 214, 8. [CrossRef]

26. Lipunov, V.; Gorbovskoy, E.; Kornilov, V.; Panasyuk, M.I.; Amelushkin, A.M.; Petrov, V.L.; Yashin, I.V.; Svertilov, S.I.; Vedenkin, N.N. SHOK—The First Russian Wide-Field Optical Camera in Space. Space Sci. Rev. 2018, 214, 6. [CrossRef]

27. Park, I.H.; Panasyuk, M.I.; Reglero, V.; Chen, P.; Castro-Tirado, A.J.; Jeong, S.; Bogomolov, V.; Brandt, S.; Budtz-Jørgensen, C.; Chang, S.H.; et al. UFFO/Lomonosov: The Payload for the Observation of Early Photons from Gamma Ray Bursts. Space Sci. Rev. 2018, 214, 14. [CrossRef]

28. Jeong, S.; Panasyuk, M.I.; Reglero, V.; Connell, P.; Kim, M.B.; Lee, J.; Rodrigo, J.M.; Ripa, J.; Eyles, C.; Lim, H.; et al. UBAT of UFFO/Lomonosov: The X-Ray Space Telescope to Observe Early Photons from Gamma-Ray Bursts. Space Sci. Rev. 2018, 214, 16. [CrossRef] 
29. Gorbovskoy, E.S.; Lipunova, G.V.; Lipunov, V.M.; Kornilov, V.G.; Belinski, A.A.; Shatskiy, N.I.; Tyurina, N.V.; Kuvshinov, D.A.; Balanutsa, P.V.; Chazov, V.V.; et al. Prompt, early and afterglow optical observations of five $\gamma$-ray bursts: GRB 100901A, GRB 100902A, GRB 100905A, GRB 100906A and GRB 101020A. MNRAS 2012, 421, 1874-1890. [CrossRef]

30. Jeong, S.; Nam, J.W.; Ahn, K.B.; Park, I.H.; Kim, S.W.; Lee, J.; Lim, H.; Brandt, S.; Budtz-Jørgensen, C.; Castro-Tirado, A.J.; et al. Slewing Mirror Telescope optics for the early observation of UV/optical photons from Gamma-Ray Bursts. Opt. Express 2013, 21, 2263-2278. [CrossRef]

31. Gaikov, G.; Jeong, S.; Agaradahalli, V.G.; Park, I.H.; Budtz-Jørgensen, C.; Castro-Tirado, A.J.; Chen, P.; Jeong, H.M.; Kim, J.E.; Kim, M.B.; et al. Slewing mirror telescope of the UFFO-pathfinder: First report on performance in space. Opt. Express 2017, 25, 29143-29154. [CrossRef]

32. Lipunov, V.; Kornilov, V.; Gorbovskoy, E.; Shatskij, N.; Kuvshinov, D.; Tyurina, N.; Belinski, A.; Krylov, A.; Balanutsa, P.; Chazov, V.; et al. Master Robotic Net. Adv. Astron. 2010, 2010, 349171. [CrossRef]

33. Kornilov, V.; Lipunov, V.M.; Gorbovskoy, E.S.; Belinski, A.A.; Kuvshinov, D.A.; Tyurina, N.V.; Shatsky, N.I.; Sankovich, A.V.; Krylov, A.V.; Balanutsa, P.V.; et al. Robotic optical telescopes global network MASTER II. Equipment, structure, algorithms. Exp. Astron. 2012, 33, 173-196. [CrossRef]

34. Pruzhinskaya, M.V.; Krushinsky, V.V.; Lipunova, G.V.; Gorbovskoy, E.S.; Balanutsa, P.V.; Kuznetsov, A.S.; Denisenko, D.V.; Kornilov, V.G.; Tyurina, N.V.; Lipunov, V.M.; et al. Optical polarization observations with the MASTER robotic net. New Astron. 2014, 29, 65-74. [CrossRef]

35. Lipunov, V.; Gorbovskoy, E.; Kornilov, V.; Krushinskiy, V.; Vlasenko, D.; Tiurina, N.; Balanutsa, P.; Kuznetsov, A.; Budnev, N.; Gress, O.; et al. MASTER Optical Polarization Variability Detection in the Microquasar V404 CYG/GS 2023+33. ApJ 2016, 833, 198-209. [CrossRef]

36. Gorbovskoy, E.S.; Lipunov, V.M.; Buckley, D.A.H.; Kornilov, V.G.; Balanutsa, P.V.; Tyurina, N.V.; Kuznetsov, A.S.; Kuvshinov, D.A.; Gorbunov, I.A.; Vlasenko, D.; et al. Early polarization observations of the optical emission of gamma-ray bursts: GRB 150301B and GRB 150413A. MNRAS 2016, 455, 3312-3318. [CrossRef]

37. Sakamoto, T.; Yoshida, A.; Kawakubo, Y.; Moriyama, M.; Yamada, Y.; Yamaoka, K.; Nakahira, S.; Takahashi, I.; Asaoka, Y.; Ozawa, S.; et al. GRB 160908A: CALET Gamma-Ray Burst Monitor detection. GCN Circ. 2016, 19903, 1.

38. Amelushkin, A.M.; Barinova, V.O.; Bogomolov, A.V.; Bogomolov, V.V.; Iyudin, A.F.; Kalegaev, V.V.; Panasyuk, M.I.; Petrov, V.L.; Svertilov, S.I.; Yashin, I.V.; et al. GRB 160720A: Lomonosov BDRG gamma ray detection and MASTER limit. GCN Circ. 2016, $19728,1$.

39. Dzhioeva, N.L.; Amelushkin, A.M.; Barinova, V.O.; Bogomolov, A.V.; Bogomolov, V.V.; Iyudin, A.F.; Kalegaev, V.V.; Panasyuk, M.I.; Petrov, V.L.; Svertilov, S.I.; et al. GRB 160824B: Lomonosov BDRG gamma ray detection and MASTER limit. GCN Circ. 2016, 19884, 1.

40. Lipunov, V.M.; Sadovnichy, V.A.; Panasyuk, M.I.; Yashin, I.V.; Svertilov, S.I.; Svinkin, D.; Gorbovskoy, E.; Simakov, S.G.; Topolev, V.; Lipunova, G.V.; et al. Possible Traces of Long Gamma Ray Burst Three Stage Collapse From Prompt Multiwavelength Observations of GRB160625B. Astron. Astrophys. 2021. (under review).

41. Sadovnichy, V.; Panasyuk, M.; Svertilov, S.I.; Lipunov, V.M.; Bogomolov, V.V.; Gorbovskoy, E.S.; Bogomolov, A.V.; Castro-Tirado, A.J.; Gabovich, A.; Hu, Y.; et al. Prompt and Follow-up Multi-wavelength Observations of the GRB 161017A. ApJ 2018, 861, 48-59. [CrossRef]

42. Meegan, C.; Lichti, G.; Bhat, P.N.; Bissaldi, E.; Briggs, M.S.; Connaughton, V.; Diehl, R.; Fishman, G.; Greiner, J.; Hoover, A.S.; et al. The Fermi Gamma-Ray Burst Monitor. ApJ 2009, 702, 791-804. [CrossRef]

43. Burns, E. on behalf of the Fermi GBM Team. GRB 160625B: Fermi GBM initial observations. GCN Circ. 2016, $19581,1$.

44. Dirirsa, F.; Racusin, J.; McEnery, J.; Desiante, R. on behalf of the Fermi-LAT team. GRB 160625B: Fermi-LAT detection of a bright burst. GCN Circ. 2016, 19580, 1.

45. Lipunov, V.; Gorbovskoy, E.; Tyurina, N.; Kornilov, V.; Balanutsa, P.; Kuznetsov, A.; Kuvshinov, D.; Rebolo, R.; Serra, M.; Lodieu, N.; et al. GRB 160625B: Global MASTER-Crimea prompt optical emission detection. GCN Circ. 2016, $19683,1$.

46. Evans, P.A. on behalf of the Swift team. GRB 160625B: Tiled Swift observations. GCN Circ. 2016, $19582,1$.

47. Melandri, A.; D'Avanzo, P.; D'Elia, V.; Burrows, D.N.; Roegiers, T.G.R.; McCauley, L.M.; Gibson, S.L.; Osborne, J.P.; Evans, P.A. on behalf of the Swift-XRT team. on behalf of the Swift-XRT team. GRB 160625B: Swift-XRT afterglow detection. GCN Circ. 2016, 19585, 1.

48. Troja, E.; Burrows, D.N.; D’Avanzo, P.; Gehrels, N.; Kennea, J.A.; Kuin, N.P.M.; Lien, A.Y.; Markwardt, C.B.; Marshall, F.E.; Melandri, A.; et al. GRB 161017A: Swift detection of a burst with a bright optical afterglow. GCN Circ. 2016, $20064,1$.

49. Svinkin, D.; Golenetskii, S.; Aptekar, R.; Frederiks, D.; Oleynik, P.; Ulanov, M.; Tsvetkova, A.; Lysenko, A.; Kozlova, A.; Cline, T. on behalf of the Konus-Wind team. Konus-Wind observation of GRB 160625B. GCN Circ. 2016, 19604, 1.

50. Oates, S.R.; D'A. A. On behalf of the Swift/UVOT team. GRB 160625B: Swift/UVOT Detection. GCN Circ. 2016, $19589,1$.

51. Troja, E.; Lipunov, V.M.; Mundell, C.G.; Butler, N.R.; Watson, A.M.; Kobayashi, S.; Cenko, S.B.; Marshall, F.E.; Ricci, R.; Fruchter, A.; et al. Significant and variable linear polarization during the prompt optical flash of GRB 160625B. Nature 2017, 547, 425-427. [CrossRef]

52. Ravasio, M.E.; Oganesyan, G.; Ghirlanda, G.; Nava, L.; Ghisellini, G.; Pescalli, A.; Celotti, A. Consistency with synchrotron emission in the bright GRB 160625B observed by Fermi. Astron. Astrophys. 2018, 613, A16. [CrossRef] 
53. Ravasio, M.E.; Ghirlanda, G.; Nava, L.; Ghisellini, G. Evidence of two spectral breaks in the prompt emission of gamma-ray bursts. Astron. Astrophys. 2019, 625, A60. [CrossRef]

54. Zhang, B.-B.; Zhang, B.; Castro-Tirado, A.J.; Dai, Z.G.; Tam, P.-H.T.; Wang, X.-Y.; Hu, Y.-D.; Karpov, S.; Pozanenko, A.; Zhang, F.-W.; et al. Transition from fireball to Poynting-flux-dominated outflow in the three-episode GRB 160625B. Nat. Astron. 2018, 2, 69-75. [CrossRef]

55. Strausbaugh, R.; Butler, N.; Lee, W.H.; Troja, E.; Watson, A.M. Evidence for a Bright-edged Jet in the Optical/Near-infrared Afterglow of GRB 160625B. ApJ Lett. 2019, 873, L6. [CrossRef]

56. Alexander, K.D.; Laskar, T.; Berger, E.; Guidorzi, C.; Dichiara, S.; Fong, W.; Gomboc, A.; Kobayashi, S.; Kopac, D.; Mundell, C.G.; et al. A Reverse Shock and Unusual Radio Properties in GRB 160625B. ApJ 2017, 848, 69. [CrossRef]

57. Kangas, T.; Fruchter, A.S.; Cenko, S.B.; Corsi, A.; de Ugarte Postigo, A.; Pe'er, A.; Vogel, S.N.; Cucchiara, A.; Gompertz, B.; Graham, J.; et al. The late-time afterglow evolution of long gamma-ray bursts GRB 160625B and GRB 160509A. ApJ 2020, 894, 43. [CrossRef]

58. Nakahira, S.; Yoshida, A.; Sakamoto, T.; Kawakubo, Y.; Moriyama, M.; Yamada, Y.; Yamaoka, K.; Takahashi, I.; Asaoka, Y.; Ozawa, S.; et al. GRB 160625B CALET Gamma-Ray Burst Monitor detection. GCN Circ. 2016, 19617, 1.

59. Troja, E.; Butler, N.; Watson, A.M.; Kutyrev, A.; Lee, W.H.; Richer, M.G.; Fox, O.; Prochaska, J.X.; Bloom, J.; Cucchiara, A.; et al. GRB 160625B: RATIR Optical and NIR Afterglow Detection. GCN Circ. 2017, 19588, 1.

60. Gorbovskoy, E.; Lipunov, V.; Tyurina, N.; Kornilov, V.; Balanutsa, P.; Kuznetsov, A.; Kuvshinov, D.; Rebolo, R.; Serra, M.; Lodieu, N.; et al. GRB 160625B: Global MASTER-Net prompt optical emission detection. GCN Circ. 2016, 19612, 1.

61. Lipunov, V.; Vladimirov, V.V.; Gorbovskoi, E.S.; Kuznetsov, A.S.; Zimnukhov, D.S.; Balanutsa, P.V.; Kornilov, V.G.; Tyurina, N.V.; Gress, O.A.; Vlasenko, D.M.; et al. The Concept of a Multi-Functional Astronomy Complex and Dynamically Integrated Database Applied to Multi-Channel Observations with the MASTER Global Network. Astron. Rep. 2019, 63, 293-309. [CrossRef]

62. Barthelmy, S.D. Observing strategies using GCN. AIP Conf. Proc. 1998, 428, 129.

63. Barthelmy, S.D.; Butterworth, P.; Cline, T.L.; Gehrels, N.; Marshall, F.; Takeshima, T.; Connaughton, V.; Kippen, R.M.; Kouveliotou, C.; Robinson, C.R. The GRB coordinates network (GCN): A status report. AIP Conf. Proc. 1998, 428, 99.

64. Yurkov, V.; Sergienko, Y.; Varda, D.; Sinyakov, E.; Gorbovskoy, E.; Lipunov, V.; Kornilov, V.; Kuvshinov, D.; Tyurina, N.; Balanutsa, P.; et al. GRB161017.74 MASTER-Net OT detection. GCN Circ. 2016, $20063,1$.

65. Vestrand, W.T.; Wozniak, P.R.; Wren, J.A.; Fenimore, E.E.; Sakamoto, T.; White, R.R.; Casperson, D.; Davis, H.; Evans, S.; Galassi, M.; et al. A link between prompt optical and prompt $\gamma$-ray emission in $\gamma$-ray bursts. Nature 2005, 435, 178-180. [CrossRef] [PubMed]

66. Lipunov, V.; Simakov, S.; Gorbovskoy, E.; Vlasenko, D. Smooth Optical Self-similar Emission of Gamma-Ray Bursts. ApJ 2017, 845, 52. [CrossRef]

67. Cowen, D.F. On behalf of the IceCube Collaboration. IceCube 160806A EHE Neutrino Candidate Event. GCN Circ. 2016, $19787,1$.

68. Panasyuk, M.I.; Svertilov, S.I.; Bogomolov, A.V.; Bogomolov, V.V.; Amelushkin, A.M.; Barinova, V.O.; Iyudin, A.F.; Kalegaev, V.V.; Nguen, D.; Petrov, V.L.; et al. IceCube 160806A: Lomonosov BDRG gamma ray prompt observations. GCN Circ. 2016, $19791,1$. 